\title{
Detection of shape orientation depends on salient axes of symmetry and elongation: Evidence from visual search
}

\author{
LUC BOUTSEN \\ University of Birmingham, Birmingham, England \\ and \\ CHRISTIAN MARENDAZ \\ Université Pierre Mendès France, Grenoble, France
}

\begin{abstract}
Three experiments investigated the role of the global spatial structure of two-dimensional (2-D) shapes in terms of symmetry and elongation on visual search for shape orientation. Experiment 1 demonstrated the often reported orientation search asymmetry (i.e., a faster detection of a tilted target among vertical distractors than the reverse) for the global orientation of 2-D polygons that possess a salient, "principal" axis of symmetry or elongation. Moreover, the search asymmetry depended on the orientation of the principal axis, rather than on the orientation of local contours. Further exploration of this effect with polygons (Experiment 2) showed that the search asymmetry for global orientation occurred for shapes containing an axis of symmetry; elongation, on the other hand, did not seem to be crucial. Finally, Experiment 3 demonstrated orientation search asymmetries with shapes composed of curved rather than straight contours: Here, the search asymmetry occurred as a function of the orientation of both axes of symmetry and elongation. Overall, search for global orientation was less efficient than search for local orientation. The results suggest that the perception of the global orientation of shapes is mediated by axis-based descriptions in terms of perceptually salient axes of symmetry and elongation.
\end{abstract}

In order to interact appropriately with objects in the three-dimensional (3-D) world, an observer must take into account a variety of visual attributes of objects, such as location, shape, size, and orientation. This imposes specific requirements on the processing characteristic of the observer's visual system. By computing object attributes on the basis of retinal image information, efficient motor responses toward objects can be prepared and executed (e.g., Jeannerod, 1997). Moreover, before object-oriented actions can take place, the visual system must detect certain object attributes sufficiently early in the time course of visual processing in order to select target objects. This requirement seems to be met, since there is evidence that visual processing proceeds broadly in two stages (e.g., Duncan \& Humphreys, 1989; Neisser, 1967; Treisman, 1985; Treisman \& Gelade, 1980; Wolfe, 1994). In a preattentive stage, a number of object attributes, such as color, motion, and

This research was conducted by L.B. in partial fulfillment of the requirements for a PhD at the Université Pierre Mendès France, Grenoble, France. Portions of the data were presented at the 20th European Conference on Visual Perception, Helsinki (August, 1997). This research was funded by the Université Pierre Mendès France, the National Centre of Scientific Research (Grant CNRS UMR 5105), and the Région Rhône-Alpes. The authors thank Gordon C. Baylis, Marisa Carrasco, Todd Horowitz, and Steve Yantis for comments on a previous version of the manuscript. Correspondence should be addressed to L. Boutsen, School of Psychology, University of Birmingham, Edgbaston, Birmingham B15 2TT, U.K. (e-mail: 1.boutsen@bham.ac.uk). orientation, are processed rapidly across the visual field without the employment of focal attention. The output of preattentive processing of the visual scene is a rather fragmented representation of the extracted features listed in different retinotopic maps; further processing is needed to construct a more coherent representation of the visual scene in which objects can be identified. This is accomplished in an attentive stage, in which the allocation of focal attention to portions of the visual field subserves the combination of features into rich representations of objects, in which, for example, the spatial relations between different features of an object are established. Although there is some consensus about the existence of preattentive and attentive processing stages in vision (Wolfe, 1998), there is less agreement about what visual information is extracted at the different stages. Recent studies have shown that preattentive processing of visual information is not limited to basic features, such as color, size, motion, and line orientation, but extends to more complex features, such as 3-D orientation (Enns \& Rensink, 1990, 1991), 3-D surfaces (Sun \& Perona, 1996), and illusory contours (Davis \& Driver, 1994). There is also evidence that the representation of the (image-plane) orientation of objects is more complex than previously assumed. In the present study, we addressed this issue with respect to the processing of the orientation of two-dimensional (2-D) shapes.

Many studies aimed at identifying basic visual features use the visual search paradigm. In a visual search 
task, observers are presented with a display containing multiple elements, and they have to indicate the presence of a (usually predefined) target element among a variable number of distractor elements. The extent to which reaction times (RTs) increase as a function of the total number of elements in the display (as evidenced by search slopes) is indicative of the efficiency with which the visual features that differentiate between the target and distractors are processed (e.g., Treisman \& Souther, 1985; Wolfe, 1998). If RTs are relatively unaffected by the number of elements, or set size (e.g., search slopes below $10 \mathrm{msec} / \mathrm{item}$ ), then it is concluded that the distinctive target feature is processed efficiently, and possibly in parallel, across the visual field. In contrast, large increases in RT as a function of set size suggest that the detection of the distinctive feature requires the employment of selective attention through scanning of the display in a more serial manner. Although efficient and less efficient search functions have been interpreted in terms of two distinct search mechanisms, one parallel and the other serial (e.g., Treisman \& Gelade, 1980; Wolfe, 1994), this dichotomy has been criticized on theoretical and empirical grounds (e.g., Chelazzi, 1999; Itti \& Koch, 2000; McElree \& Carrasco, 1999; Townsend, 1972, 1990; Wolfe, 1998). For example, set-size effects (i.e., increases in RT as a function of the number of elements) depend on attentional factors and on sensory factors (Carrasco, McLean, Katz, \& Frieder, 1998; Carrasco \& Yeshurun, 1998; Cheal \& Lyon, 1992; J. Palmer, 1994, 1995) and can be accounted for by parallel limited-capacity processes (e.g., Duncan \& Humphreys, 1989; McElree \& Carrasco, 1999). Parallel limited-capacity models may also account for search asymmetries, if one restates the efficiency of target detection in terms of discriminability and signal-to-noise ratio (e.g., McElree \& Carrasco, 1999; J. Palmer, 1995; Shaw, 1984). Alternatively, one can assume that visual search performance may reflect the operation of different parallel and serial processing modules, with search being more efficient in the former stages than in the latter stages (Wolfe, 1998). While a discussion of this debate is beyond the scope of the present study, it is this qualitative and rather neutral interpretation of visual search performance in terms of efficiency that we will adopt. Under this scheme, "efficient" search performance is assumed to be both fast and accurate and less affected by attentional demands or sensory factors than is "inefficient" search.

The visual search paradigm has been a useful tool in the identification of visual features, such as color, size, motion, and orientation, that seem to be extracted at early stages in visual processing (for a review, see Wolfe, 1998). We will now review studies of the processing of local and global orientation.

\section{Representation of Local Orientation}

Studies of orientation processing generally fall into two classes: local and global orientation. The first contains numerous studies that test detection of the orientation of the simplest possible oriented stimuli-that is, lines (defined by luminance contrast) — which have an unambiguous explicit or local orientation. These studies have revealed several basic findings. First, processing of line orientation in visual search or detection tasks is effortless, provided the orientations of the target and distractors are sufficiently different (e.g., Foster \& Ward, 1991a, 1991b; Marendaz, Stivalet, \& Genon, 1991; Nothdurft, 1991). Second, orientation acuity is better for vertical or horizontal orientations than for oblique orientations, suggesting that horizontal and vertical are treated as reference orientations (Appelle, 1972; Heeley, Buchanan-Smith, Cromwell, \& Wright, 1997; Orban, Vandenbussche, \& Vogels, 1984; Vogels \& Orban, 1985). Third, for orientations deviating by a small amount (about $20^{\circ}$ ) from the vertical, search for oriented lines shows an asymmetry: Search for a tilted target among vertical distractors is fast and accurate, whereas search for a vertical target among tilted distractors is slow and inefficient (Carrasco et al., 1998; Cavanagh, Arguin, \& Treisman, 1990; Foster \& Ward, 1991a, 1991b; Foster \& Westland, 1995; Marendaz, 1998; Marendaz, Stivalet, Barraclough, \& Walkowiac, 1993; Marendaz et al., 1991; Meigen, Lagreze, \& Bach, 1994; Treisman, 1985; Treisman \& Gormican, 1988). A similar orientation search asymmetry (henceforth, orientation asymmetry) has been observed for small deviations from the horizontal (Marendaz et al., 1991). These findings have led to models of orientation processing based on the coding of local orientation with respect to two reference orientations, vertical and horizontal (Foster \& Ward, 1991a, 1991b; Treisman \& Gormican, 1988; Westland \& Foster, 1995; Wolfe, 1994). For example, the model by Foster and his colleagues uses spatial filtering of the image at principal orientations in order to explain orientation asymmetries.

\section{Representations of Global Orientation}

Recent studies of orientation processing have used stimuli whose orientation is defined in different media such as texture, motion, or binocular disparity (e.g., Bravo \& Blake, 1990; Cavanagh et al., 1990; Li \& Westheimer, 1997; Saarinen, 1994; Westheimer \& Li, 1996), and configurations of contours possessing a global (2-D or 3-D) orientation (e.g., Bilsky \& Wolfe, 1995; Enns \& Rensink, 1990, 1991; Found \& Müller, 1997). Because the orientation of these more complex stimuli cannot be reduced to the orientation of local contours, as in the case of line segments, their orientation is implicit (Li \& Westheimer, 1997) or global (Found \& Müller, 1997). The suggestion that global orientation is processed early in vision has particular implications for the proposal that the representation of orientation is merely the product of spatial image filtering. Rather, it seems that different perceptual processes, in addition to filtering, cooperate in the processing of orientation (cf. Grossberg, Mingolla, \& Ross, 1994). For instance, global orientation can emerge as a function of the grouping of elements (Bravo \& Blake, 1990; see Figure 1A) and can be processed at least as fast as local orientation (Saarinen, 1994; see Figure 1B). Compatible with the results of these studies is the finding that 
A
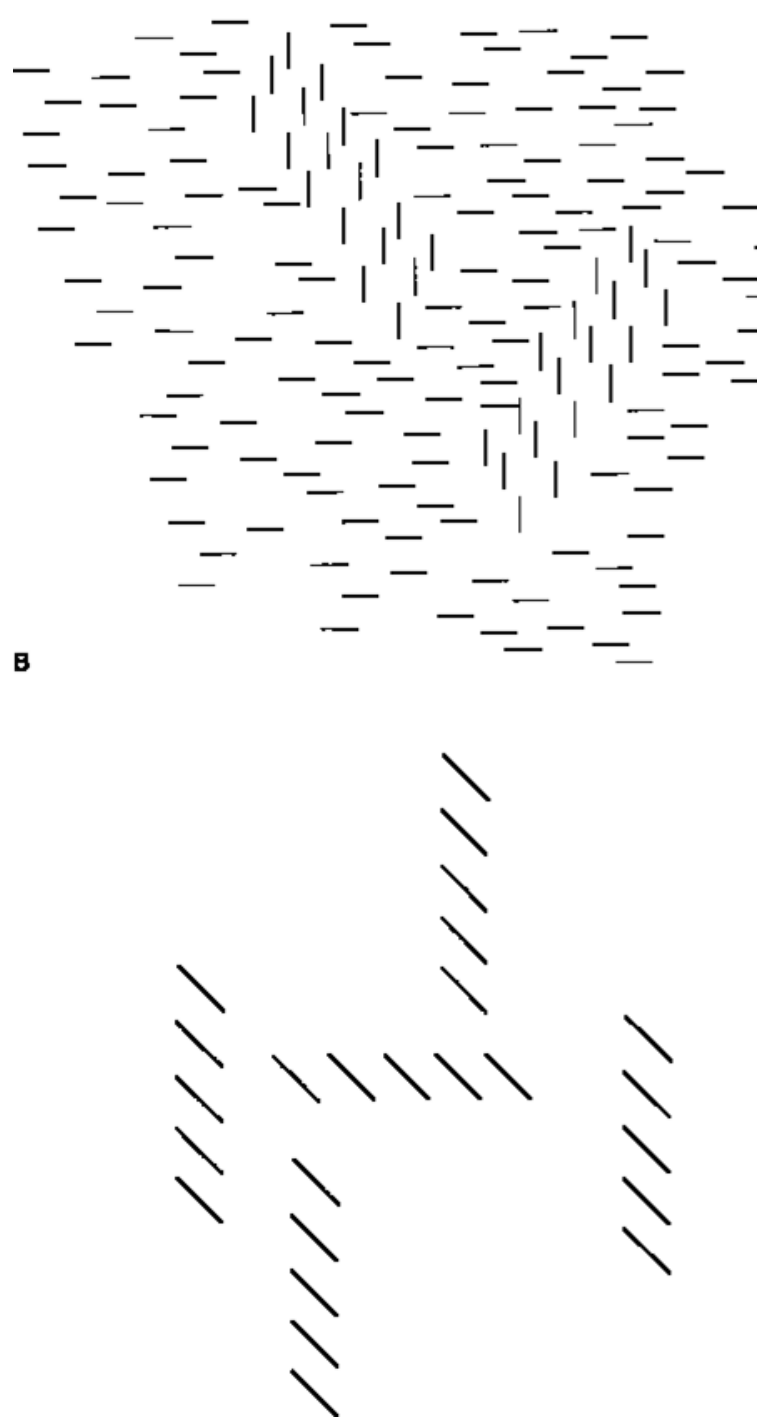

Figure 1. Example displays used by (A) Bravo and Blake (1990) and (B) Saarinen (1994). In both displays, target and distractor configurations differ in global orientation but not in local orientation.

irrelevant global orientation can disrupt the detection of local orientation (Found \& Müller, 1997, Experiment 5; Lauwereyns \& d'Ydewalle, 1997).

Other evidence for more complex representations of orientation comes from studies demonstrating efficient detection of the orientation of configurations of connected contours. For example, Enns and Rensink (1990) demonstrated that the detection of such configurations was efficient if the target had a clear 3-D interpretation (see Figure 2A). These authors reasoned that preattentive processes rapidly recover conjunctions of contours that indicate 3-D surface orientation (e.g., Y-junctions), whereas junctions that do not signal 3-D orientation(e.g., $\mathrm{T}$-junctions) are not processed in the same manner. This interpretation is consistent with the proposal that the visual system detects "nonaccidental" properties in an image in order to construct representations of 3-D objects (Biederman, 1987; Kanade, 1981).

Of particular relevance to the present study is work by Found and Müller (1997), who examined the role of local and global orientation in visual search. In a series of experiments, they demonstrated that search for the global orientation of configurations of contours (Figure 2B) was very efficient, provided the stimuli had an unambiguous global orientation. Search was also unaffected when the shapes were defined by contours or by texture, therefore ruling out a spatial filtering account of global orientation detection (cf. Bravo \& Blake, 1990; Cavanagh et al., 1990). Finally, Found and Müller (1997, Experiment 6) demonstrated a search asymmetry for the global orientation of configurations of contours: Search for a vertical diamond (Figure 2B) among slightly tilted (about $18^{\circ}$ ) diamonds was less efficient than the reverse, as was evidenced by slower RTs and steeper search slopes for vertical targets. This indicates that search was based on global shape orientation, rather than on the orientation of local contours. Consistent with this, the authors described the global orientation as "axial," which suggests that the assignment of an orientation to a shape is accomplished by deriving an implicit axis on the basis of contour information (cf. Howard, 1982). Thus, the orientation asymmetry for diamonds depends on the orientation of the implicit axis, and the dectection of global orientation then comes down to the task of deriving an axis on the basis of contour information. This interpretation was further supported by the results of another experiment (Found \& Müller, 1997, Experiment 1), in which search for global orientation was impeded when the configuration did not have an unambiguous axial orientation, even when the target possessed a distinctive local contour relative to the distractors. Thus, ambiguity in the derivation of an orientation axis seems to impede orientation search.

On the basis of these findings, the question arises as to how an unambiguous axis is derived from shape contour. As Found and Müller (1997) noticed, the relation between local (contour) and global (shape) orientation is not straightforward: The same set of contours can be assembled into configurations with different orientations. Any processing of local orientations on the basis of spatial filtering would be insufficient by itself to derive a global orientation. Therefore, additional perceptual processes operating on the ensemble of contours seems to be required. One possible process involves computing an axis on the basis of salient characteristics of the global spatial structure of contours. In the context of computational models of image processing, it has been proposed that the global spatial structure of shape is described in terms of elongation and bilateral symmetry (Biederman, 1987; Blum \& Nagel, 1978; Marr \& Nishihara, 1978). According to Marr (1982), shape elongation and symmetry define axes that provide a reference frame for describing parts or contours of shapes and their spatial relations. 
A

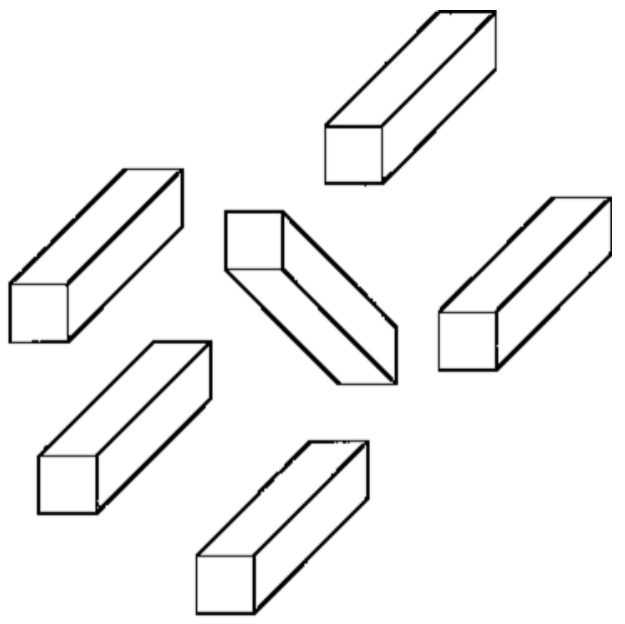

B

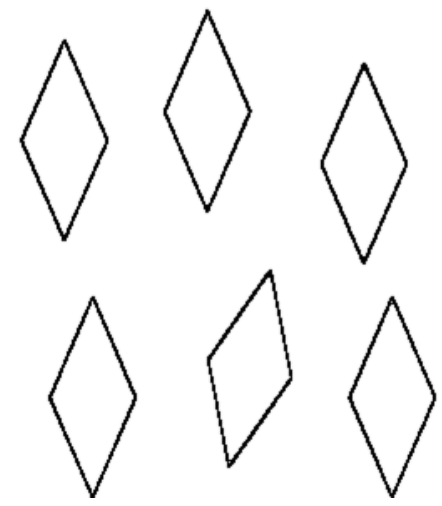

Figure 2. Example displays used by (A) Enns and Rensink (1990) and (B) Found and Müller (1997).

The resulting shape description is relatively invariant for changes in orientation, since the contours are described relative to an axis centered on the shape.

By now, evidence exists for the role of axis-based descriptions in the perception of 2-D shapes (Humphreys, 1983, 1984; Humphreys \& Quinlan, 1988; S. E. Palmer, 1980, 1985; Quinlan, 1991; Quinlan \& Humphreys, 1993; Sekuler, 1996). For example, shape matching studies (Humphreys \& Quinlan, 1988; Quinlan \& Humphreys, 1993) have shown that shapes possessing an unambiguous principal axis yield the same description across a variety of orientations, whereas shapes without a salient principal axis can yield different descriptions depending on their orientation. Also, with the polygons shown in Figure 3, Quinlan and Humphreys (1993) showed that observers were more consistent in drawing an axis of symmetry than in drawing an axis of elongation or an axis in an asymmetrical shape. Thus, global shape orientation can be based on the detection of a salient principal axis of symmetry and elongation.

Some of the results from Found and Müller's (1997) study hint at a role for axes of elongation and symmetry in the perception of global orientation. In their Experiments 1 and 6, search was more efficient for shapes that were symmetrical and elongated along the same axis. Other evidence for the role of symmetry in orientation discrimination comes from $\mathrm{Li}$ and Westheimer (1997), who showed that orientation acuity was equally accurate for lines as for symmetrical contours. Acuity decreased, however, for asymmetrical contours, indicating that the symmetry axis of the configuration was used in orientation discrimination. Finally, studies demonstrating detection of symmetry at vertical orientation that is superior to detection of symmetry at other orientations provide convergent evidence for a relation between symmetry and orientation (e.g., Corballis \& Roldan, 1975; Mach, 1886/1959; S. E. Palmer \& Hemenway, 1978; Pashler, 1990; Wagemans, Van Gool, \& d'Ydewalle, 1992; Wenderoth, 1994).

The purpose of the present study was to further examine the role of axes of symmetry and elongation in the detection of the global orientation of 2-D shapes. ${ }^{1}$ We examined, in three experiments, search asymmetries for the global orientation of shapes with designated axes of symmetry and elongation (see Table 1). Specifically, we focused on three issues. In Experiment 1, we replicated Found and Müller's (1997, Experiment 6) demonstration of an orientation asymmetry for shapes with an unambiguous axis of symmetry and elongation in vertical and oblique orientations, and we examined search performance for horizontal and oblique orientations. Also, control experiments involving search for local orientation were conducted with stimuli consisting of conjunctions of contours. Second, we separately assessed the role of axes of symmetry and elongation in search for global orientation (Experiment 2). If axes of symmetry and elongation determine global shape orientation, then the orientation
CONJOINT

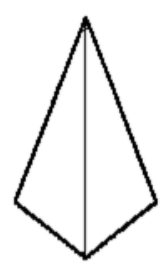

ASYMMETRICAL

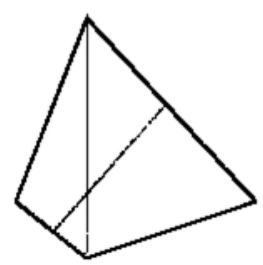

SYMMETRICAL

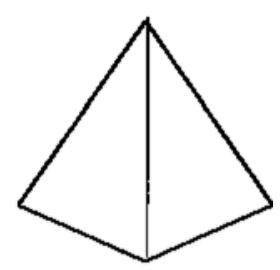

ELONGATED

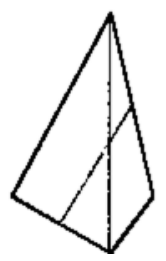

Figure 3. The polygons used in Experiments 1 and 2. The vertical line within each shape indicates the designated principal axis; these lines were not presented in the experiments. 
Table 1

Overview of Experiments 1-3: Stimulus Type and Orientations

\begin{tabular}{cll}
\hline Experiment & Stimulus Type & \multicolumn{1}{c}{ Orientations } \\
\hline 1A & Conjoint shape & Vertical-oblique \\
1B & Conjoint shape & Horizontal-oblique \\
1C & Conjoint left side & Vertical-oblique \\
& Conjoint right side & Vertical-oblique \\
2A & Asymmetrical & Vertical-oblique \\
2B & Symmetrical & Vertical-oblique \\
2C & Elongated & Vertical-oblique \\
3A & Conjoint curve & Vertical-oblique \\
3B & Elongated curve & Vertical-oblique \\
\hline
\end{tabular}

Note-Each orientation was assigned to targets and distractors. The orientations are labeled according to the orientation of the designated principal axis in each shape (see Figure 3).

asymmetry should be reduced for shapes with no salient axis of symmetry or elongation. Third, we examined whether the orientation asymmetry extended to configurations of curved contours, possessing a salient axis of symmetry and/or elongation (Experiment 3 ). The observation of an orientation asymmetry with curved contours would further support the proposal that visual search for global orientation is based on the detection of the orientation of a principal axis, rather than on the orientation of local contours.

\section{GENERAL METHOD}

Throughout Experiments 1-3, the following method was adopted.

\section{Participants}

One hundred twenty-eight undergraduate students in psychology from the Université Pierre Mendès France in Grenoble participated in the experiments for course credit. Each student participated in only one experiment. All participants had normal or corrected-tonormal vision, and they were not aware of the purpose of the experiments.

\section{Stimuli and Apparatus}

The experiments were controlled by an IBM-compatible computer. The stimuli were presented on a 15-in. SVGA monitor, with a vertical refresh rate of $60 \mathrm{~Hz}$ and in an $800 \times 600$ graphics mode.

Stimulus displays consisted of polygons (Experiments 1 and 2) or curved contours (Experiment 3). Examples of the displays are shown in Figures 4, 6, and 8 for Experiments 1, 2, and 3, respectively. The polygons in Experiments 1 and 2 had the same shape as a subset of the stimuli used by Quinlan and Humphreys (1993). In accordance with these authors, the configuration of the implicit axes for each shape was defined in terms of the distance between two opposite vertices. This resulted in each shape having two orthogonal axes. Four different polygons resulted from the orthogonal combination of bilateral symmetry and elongation (Figure 3): The conjoint shape had an axis of symmetry aligned with an axis of elongation. The symmetrical and elongated shapes possessed, respectively, an axis of symmetry and elongation only (thus, the symmetrical shape was not elongated, and the elongated shape was asymmetrical). The asymmetrical shape did not possess an axis of symmetry or of elongation.

Shape elongation was determined by aspect ratio- that is, the ratio between the length of the vertical axis (the length of the shape) relative to the length of the horizontal axis (the maximal width of the shape). The aspect ratio of the elongated shapes (i.e., the conjoint and elongated shapes) was 1.5 , whereas that of shapes without elongation was 1 (cf. the symmetrical and asymmetrical shapes). Finally, two additional shapes were used, an elongated curve and a conjoint curve (Figure 8). These shapes both contained an axis of elongation. The elongated curve was asymmetrical, whereas the conjoint curve was symmetrical around the axis of elongation. The symmetry of the conjoint shape was created by mirror-reflecting the side to the left of the main axis of the elongated curve. The aspect ratios of the conjoint and elongated curves were 1.5 and 2, respectively (the larger aspect ratio of the elongated curve was due to its smaller width relative to the conjoint curve).

The stimuli were presented as high-contrast luminance-defined contours (of 1 pixel, $0.006^{\circ}$ of visual angle) in white on a dark background (i.e., shapes were not filled). The shapes subtended maximally $1.5^{\circ} \times 1^{\circ}$ of visual angle. At a viewing distance of $110 \mathrm{~cm}$, the contours appeared smooth.

Stimulus displays consisted of the elements of the same shape in one orientation (target-absent displays) or two orientations (targetpresent displays): horizontal (Experiment 1B) or vertical (other experiments) and oblique. Vertical shapes had their designated principal axis of elongation or symmetry aligned with the vertical, as defined in an environment-centered reference frame. (The axes are shown in Figure 3 as vertical dashed lines.) The asymmetrical shape, which contained no axis of symmetry or elongation, was presented with one axis aligned with the vertical, as shown in Figure 3. The conjoint shapes in Experiment $1 \mathrm{~B}$ were presented with the designated principal axis horizontal. Oblique shapes were rotated by approximately $18^{\circ}$ clockwise from the horizontal in Experiment $1 \mathrm{~B}$ (conjoint shapes) and $18^{\circ}$ counterclockwise from the vertical in the other experiments. As a result, the principal axes of the shapes were now oblique.

Each stimulus display consisted of a conf iguration of 4, 7, or 10 elements, with the target, if present, included. New conf igurations were generated at each trial by randomly positioning the elements on one of 16 positions within a circular aperture whose diameter subtended $10^{\circ}$ of visual angle and by adding a random jitter of maximum 30 pixels $\left(0.20^{\circ}\right.$ of visual angle) to the horizontal position and the vertical position of each element to avoid exact alignment of neighboring elements.

\section{Design}

The design of each experiment included three factors, which were manipulated within participants: target orientation, set size, and target presence. The orientation tasks (e.g., search for a vertical target or an oblique target) were blocked within participants. Set size $(4,7$, or 10 elements) and target presence were manipulated within each orientation task. The target was present on half of the trials of each orientation task.

Each experimental session consisted of two blocks of trials, corresponding to the two orientation tasks that were administered: a search for a horizontal target (Experiment 1B) or a vertical target (other experiments) among oblique distractors, and a search for an oblique target among horizontal distractors (Experiment 1B) or vertical distractors (other experiments). Within each block, each combination of three set sizes $(4,7$, and 10 items) with the target absent or present was tested 16 times in a randomized order, resulting in 96 trials per orientation block. The order of presentation of the two orientation blocks was counterbalanced between participants. Ten practice trials were administered before each block.

\section{Procedure}

At each trial, the stimulus display was preceded by a fixation point and followed by a 2 -sec blank interval after a response was made. The fixation point was presented for $1.5 \mathrm{sec}$, and the stimulus display was presented immediately following the offset of the fixation point. On each trial, the participants responded as to 
Table 2

Percent Errors in Experiments 1-3

\begin{tabular}{lccccc}
\hline & \multicolumn{4}{c}{ Target Orientation } \\
\cline { 2 - 3 } \cline { 5 - 6 } \cline { 5 - 6 } \cline { 5 - 6 } \cline { 5 - 6 } Experiment & 10.0 & 3.3 & & \multicolumn{2}{c}{ Oblique } \\
\cline { 2 - 3 } & Absent & Present & & Absent & Present \\
\hline 1A & 12.3 & 6.0 & & 10.7 & 6.0 \\
1B & & & & 3.8 \\
1C & 1.6 & 1.5 & & 12.7 & 4.2 \\
$\quad$ Left side & 5.6 & 3.3 & & 2.3 & 3.9 \\
$\quad$ Right side & 10.0 & 8.2 & & 7.3 & 4.1 \\
2A (Asymmetrical) & 9.5 & 3.9 & & 2.7 & 1.6 \\
2B (Symmetrical) & 4.9 & 5.4 & & 11.9 & 8.3 \\
2C (Elongated) & 11.6 & 2.9 & & 3.1 & 0.5 \\
3A (Conjoint curve) & 11.8 & 3.4 & & 3.8 & 1.0 \\
3B (Elongated curve) & 11.8 &
\end{tabular}

*Except for Experiment 1B, in which the target orientation was horizontal versus oblique.

whether the target was present in the display by pressing one of two response buttons corresponding to "present" and "absent" responses. Half of the participants in each experiment pressed the right button for target-present responses and the left button for target-absent responses. This mapping was reversed for the other half of the participants. Feedback was provided following incorrect responses in practice and experimental trials by means of a low-pitched tone. On each experimental trial, RT was measured to the nearest millisecond following the response, and accuracy was measured.

The participants were tested individually. Each participant sat $110 \mathrm{~cm}$ from the screen in a darkened room. He or she looked at the screen through a view tunnel (i.e., a black-painted metal cone positioned in front of the screen) whose diameter subtended a visual angle of $10^{\circ}$, and contextual cues to orientation were eliminated as much as possible. All stimuli were viewed binocularly. The position of the participant's head was upright and stabilized by a frame and a chinrest. Detailed written instructions explained the task and showed displays including the target. It was stressed that the participants should respond as quickly as possible while minimizing errors. In order to avoid hasty responses, the computer program refused to accept responses made within $200 \mathrm{msec}$ following the onset of the stimulus. A short break was given between the two blocks. Each session took about $45 \mathrm{~min}$.

\section{Analysis of Results}

For each participant, a cutoff value set at $3 S D$ s above the mean RT on correct responses was computed. Mean RTs were computed for the remaining data per participant for each combination of target orientation, set size, and target presence. These means were entered in a repeated measures analysis of variance (ANOVA), with target orientation, set size, and target presence as within-participants variables. Parallel analyses were performed on the mean percentage of correct responses per participant per condition. These analyses either yielded no reliable effects or yielded effects that went in the same direction as for the RTs. In the latter case, there were no indications of speed-accuracy tradeoffs (except for in the analyses of Experiments 1A, 1B, and 2A, which yielded moderate correlations between RTs and accuracy of .162, .390, and .205, respectively). Since our main interest was in analyzing the RTs, and since overall error rates were small (below 9\%), we do not report the error analyses. Error rates per target orientation and target presence for the three experiments are shown in Table 2. As can be seen, in most conditions there were more errors for present trials (misses) than for absent trials (false alarms), suggesting that the participants tended to respond prior to searching through all the items in a display.

Search slopes (msec/item) were computed on the mean RTs per target orientation as a function of set size and separately for present and absent trials. Slopes were analyzed directly (through ANOVAs) as a function of target orientation and target presence. In addition, descriptive search function statistics (slopes, intercepts, and proportions of linearity, $R^{2} \mathrm{~s}$ ) were computed for the conditions in each experiment. These are shown in Table 3 for Experiment 1 and in Table 4 for Experiments 2 and 3. The mean RTs per condition across participants are presented in Figures 5, 7, and 9 for Experiments 1, 2 , and 3, respectively. For reasons of exposition, detailed statistical analyses are given after an informal presentation of the main results (for each experiment).

\section{EXPERIMENT 1}

In Experiment 1, we presented the conjoint shape whose principal axis of elongation and symmetry might determine its global orientation (cf. Found \& Müller, 1997, Experiment 6; Quinlan \& Humphreys, 1993).

In Experiments $1 \mathrm{~A}$ and $1 \mathrm{~B}$, orientation search for small deviations from, respectively, the vertical and the horizontal was tested with displays composed of conjoint shapes (Figure 4, upper panel). If search for global orientation is based on the derivation of a principal axis from contour information, then one would expect to observe an orientation asymmetry similar to that for simple lines. In Experiment 1A, we tested this hypothesis for vertical and oblique shapes. The finding of an orientation asymmetry in this experiment would also replicate Found and Müller's (1997, Experiment 6) observations with diamond

Table 3

Slope Statistics for Experiment 1

\begin{tabular}{cccc}
\hline & Intercept & Slope \\
Condition & $(\mathrm{msec})$ & $(\mathrm{msec} / \mathrm{item})$ & $R^{2}$ \\
\hline
\end{tabular}

Experiment $1 \mathrm{~A}$

$\begin{array}{cccc}\text { Absent } & & & \\ \text { Vertical } & 1,006 & 57.1 & .989 \\ \text { Oblique } & 899 & 43.3 & .936 \\ \text { Present } & & & \\ \text { Vertical } & 1,018 & 17.9 & .999 \\ \text { Oblique } & 753 & 28.2 & .961 \\ & \text { Experiment 1B } & \end{array}$

Absent

$\begin{array}{llll}\begin{array}{l}\text { Horizontal } \\ \text { Oblique }\end{array} & 1,351 & 86.5 & .918 \\ \begin{array}{l}\text { Present } \\ \text { Horizontal }\end{array} & 1,308 & 61.8 & .717 \\ \text { Oblique } & 1,111 & 30.5 & .793 \\ & 1,208 & 21.0 & .587\end{array}$

Absent

Vertical

Oblique

Experiment 1C (Left side)

Present

Vertical

Oblique

1,008

$-3.2$

28.6

.893

1,133

$-18.6$

1,097

6.3

Experiment 1C (Right side)

Absent

Vertical

Oblique

1,123

11.5

11.4

.868

Present

Vertical

Oblique

1,049

923

3.7

$-4.8$ 
Table 4

Slope Statistics for Experiments 2 and 3

\begin{tabular}{|c|c|c|c|}
\hline Condition & $\begin{array}{l}\text { Intercept } \\
(\mathrm{msec})\end{array}$ & $\begin{array}{c}\text { Slope } \\
(\mathrm{msec} / \mathrm{item})\end{array}$ & $R^{2}$ \\
\hline \multicolumn{4}{|c|}{ Experiment 2A (Asymmetrical) } \\
\hline \multicolumn{4}{|l|}{ Absent } \\
\hline Vertical & 1,197 & 54.7 & .984 \\
\hline Oblique & 1,171 & 47.0 & .922 \\
\hline \multicolumn{4}{|l|}{ Present } \\
\hline Vertical & 1,162 & 7.5 & .154 \\
\hline Oblique & 1,018 & 23.9 & .999 \\
\hline \multicolumn{4}{|c|}{ Experiment 2B (Symmetrical) } \\
\hline \multicolumn{4}{|l|}{ Absent } \\
\hline Vertical & 1,289 & 67.5 & .960 \\
\hline Oblique & 954 & 46.6 & .903 \\
\hline \multicolumn{4}{|l|}{ Present } \\
\hline Vertical & 1,124 & 39.6 & .986 \\
\hline Oblique & 892 & 21.6 & .973 \\
\hline \multicolumn{4}{|c|}{ Experiment $2 \mathrm{C}$ (Elongated) } \\
\hline \multicolumn{4}{|l|}{ Absent } \\
\hline Vertical & 1,063 & 7.6 & .883 \\
\hline Oblique & 1,113 & 27.7 & .780 \\
\hline \multicolumn{4}{|l|}{ Present } \\
\hline Vertical & 874 & -0.5 & .023 \\
\hline Oblique & 991 & 14.9 & .998 \\
\hline \multicolumn{4}{|c|}{ Experiment 3A (Conjoint curve) } \\
\hline \multicolumn{4}{|l|}{ Absent } \\
\hline Vertical & 1,065 & 92.3 & .961 \\
\hline Oblique & 844 & 43.2 & .971 \\
\hline \multicolumn{4}{|l|}{ Present } \\
\hline Vertical & 1,037 & 52.3 & .907 \\
\hline Oblique & 753 & 19.8 & .950 \\
\hline \multicolumn{4}{|c|}{ Experiment 3B (Elongated curve) } \\
\hline \multicolumn{4}{|l|}{ Absent } \\
\hline Vertical & 1,232 & 121.4 & .979 \\
\hline Oblique & 1,015 & 44.0 & .860 \\
\hline \multicolumn{4}{|l|}{ Present } \\
\hline Vertical & 1,066 & 72.7 & .994 \\
\hline Oblique & 907 & 13.8 & .891 \\
\hline
\end{tabular}

stimuli, which were structurally similar to the conjoint shapes. In Experiment 1B, the designated principal axis of the conjoint shape was oriented horizontally. Here, a different hypothesis regarding search performance could be drawn, since it is possible that the horizontal principal axis of the conjoint shape is less salient; this may be because horizontal symmetry is less salient than vertical symmetry (e.g., Corballis \& Roldan, 1975; Pashler, 1990; Wenderoth, 1994). Thus, if the perception of global orientation depends on the detection of the shape's axis of symmetry, then the orientation asymmetry should be less pronounced or should even disappear with a horizontal symmetry axis. However, if search performance mainly depends on the detection of shape elongation, then a search asymmetry similar to that found with horizontal line segments (Marendaz et al., 1991) would be expected.

In Experiment 1C, we tested for the possibility that any search asymmetry found with the conjoint shape was due to the processing of the orientation of local contours on either side of the principal axis. If detection of the orientation of the conjoint shapes was accomplished by the processing of the orientation of the global contour, then the search asymmetries for the contours on both sides of the principal axis separately should be different, because the sides to the left and the right of the axis have opposite orientations relative to the vertical (Figure 4). More specifically, we hypothesized that the search asymmetry would be in the opposite direction when the left side of the conjoint contour (which is oriented closer to the vertical when the conjoint shape is oblique) was presented separately, as opposed to the right side (whose absolute orientation is closer to the vertical when the conjoint shape is vertical).

Forty-eight students were randomly assigned to one of the following experiments: 16 students participated in Experiment 1A, 12 participated in Experiment 1B, and 20 participated in Experiment 1C (10 in each condition).

\section{Experiment 1A: Vertical Versus Oblique}

RTs are presented in the left panel of Figure 5A. As can be seen, a search asymmetry occurred as a function of target orientation: RTs were faster for oblique targets than for vertical targets, at present and absent trials. This asymmetry is similar to the one observed with vertical and oblique lines. However, there were also differences: With
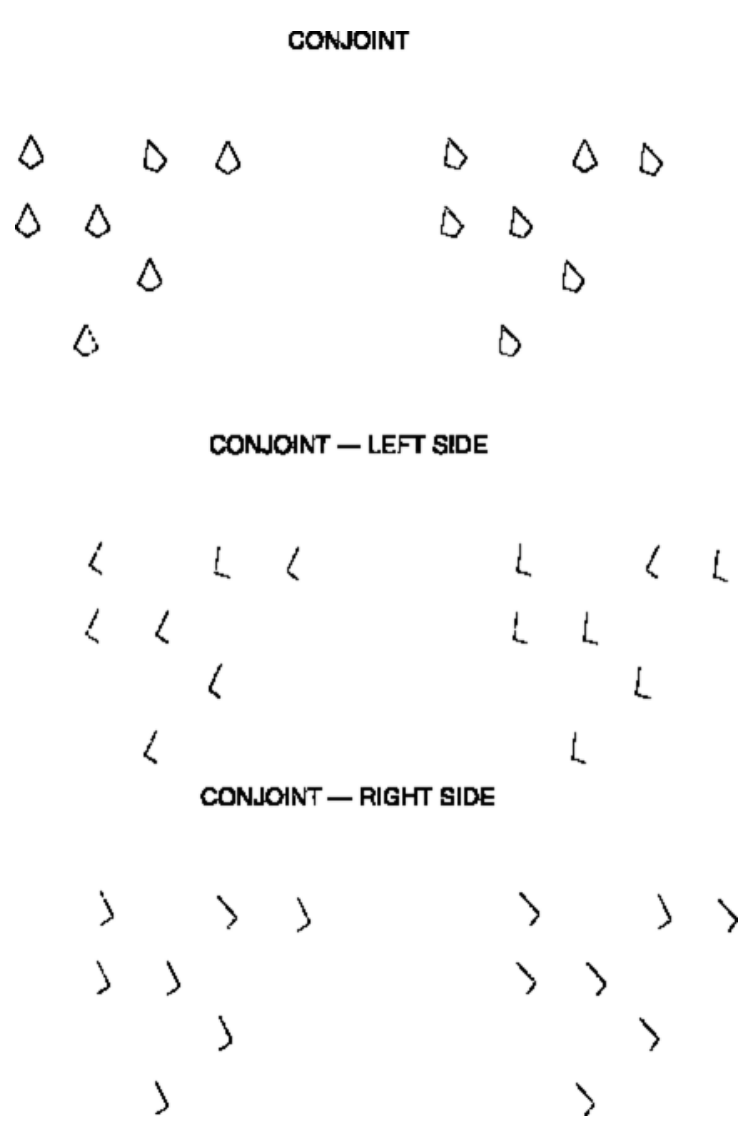

Figure 4. Example displays used in Experiment 1. The left displays show an oblique target among vertical distractors; the right displays show a vertical target among oblique distractors. 
(A)

CONJOINT: VERTICAL - OBLIOUE

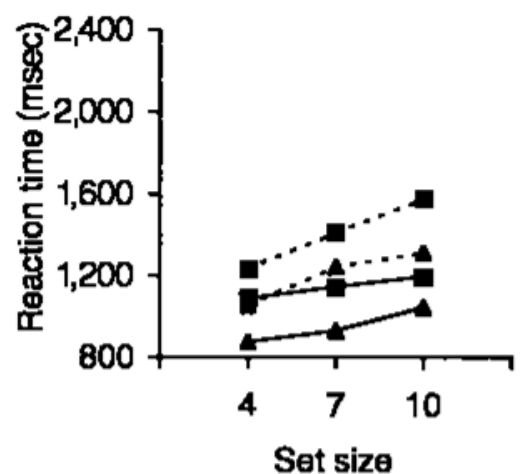

(B)

\section{CONJOINT : LEFT SIDE}

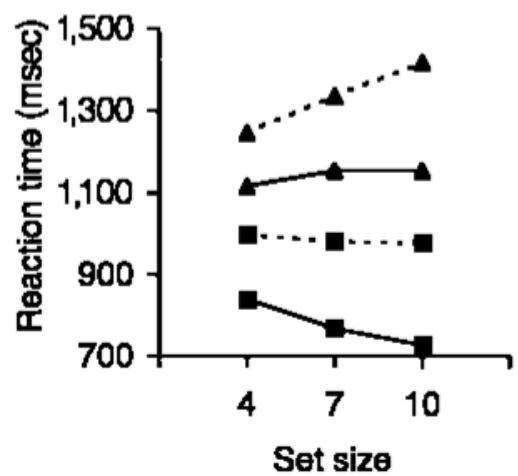

CONJOINT: HORIZONTAL - OBLIQUE
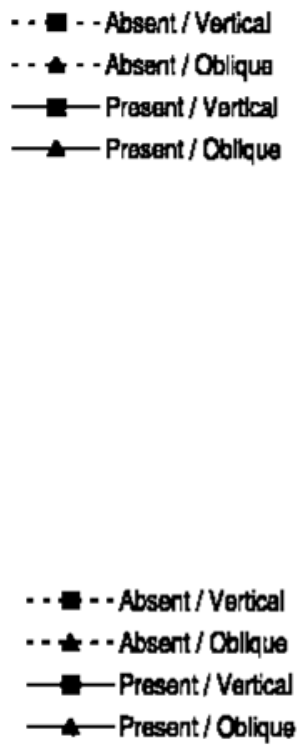

Figure 5. Mean RTs per condition in Experiment 1. The labels indicate target presence and target orientation. the conjoint shapes, RTs for vertical and oblique targets increased as a function of set size. The search slopes (Table 3) were steep, suggesting that search was inefficient. Thus, search for an oblique target was faster than for a vertical target, whereas search was less efficient than with individual lines.

RTs. The above observations were confirmed by an ANOVA on the RTs. There was a reliable effect of target orientation $\left[F(1,15)=4.51, M S_{\mathrm{e}}=420,325, p=.050\right]$ : On average, an oblique target was detected faster than was a vertical target $(1,076$ vs. $1,275 \mathrm{msec})$. There were also reliable effects of set size $\left[F(2,30)=12.01, M S_{\mathrm{e}}=\right.$ $64,459, p<.0002]$ and target presence $[F(1,15)=22.66$, $\left.M S_{\mathrm{e}}=140,014, p<.0003\right]$ : RTs increased with set size, and RTs were faster on present trials than on absent trials (Figure 5A). There was a reliable interaction between set size and target presence $\left[F(2,30)=8.08, M S_{\mathrm{e}}=14,483\right.$, $p<.002$ ], indicating that search slopes were steeper for absent trials than for present trials; there was also a reliable interaction between target orientation, set size, and target presence $\left[F(2,30)=4.82, M S_{\mathrm{e}}=6,245, p<.02\right]$. This three-way interaction was due to a steeper slope for an oblique target than for a vertical target, for present trials only. The other interactions were not reliable $\left(F_{\mathrm{S}}<1\right)$.

Slopes. An ANOVA on the search slopes yielded a reliable effect of target presence $\left[F(1,15)=8.96, M S_{\mathrm{e}}=\right.$ $1,309, p<.01]$. Search slopes were steeper for absent trials than for present trials. There was no effect of target orientation $(F<1)$. Target presence, however, interacted with target orientation $\left[F(1,15)=5.11, M S_{\mathrm{e}}=457, p<\right.$ $.04]$ : For present trials, the slope for an oblique target was steeper than for a vertical target.

\section{Experiment 1B: Horizontal Versus Oblique}

The right panel of Figure 5A shows the results of Experiment $1 \mathrm{~B}$. The orientation asymmetry completely disappeared on present trials, and it was reduced on absent trials, relative to that in Experiment 1A. Thus, at least on present trials, RTs for oblique and vertical targets were similar. Also, RTs increased with set size, and search slopes were steep, indicating inefficient search (Table 3).

RTs. An ANOVA revealed reliable effects of set size $\left[F(2,22)=15.23, M S_{\mathrm{e}}=91,717, p<.0001\right]$ and target presence $\left[F(1,11)=13.83, M S_{\mathrm{e}}=661,918, p<.004\right]$. 
Importantly, there was no reliable effect of target orientation $[F(1,11)=1.38, p>.26]$ : On average, search for an oblique target was only $89 \mathrm{msec}$ faster than for a horizontal target $(1,547 \mathrm{vs} .1,636 \mathrm{msec})$. Target orientation interacted with target presence $\left[F(1,11)=5.89, M S_{\mathrm{e}}=\right.$ $85,582, p<.04]$, indicating that RTs to horizontal and oblique targets were the same at present trials $(1,354$ and $1,324 \mathrm{msec}$ ) but that there was an advantage for horizontal distractors, relative to oblique distractors, at absent trials $(1,740$ vs. $1,947 \mathrm{msec}$ ). Search slopes (Table 3 ) were steeper for absent trials than for present trials, as was evidenced by an interaction of set size with target presence $\left[F(2,22)=12.51, M S_{\mathrm{e}}=24,594, p<.0003\right]$. The other interactions were not reliable [target orientation $\times$ target presence, $F(2,22)=1.81, p>.18$; the highest order interaction, $F<1$ ].

Slopes. An ANOVA revealed a reliable effect of target presence $\left[F(1,11)=14.79, M S_{\mathrm{e}}=2,079, p<.003\right]$, confirming that slopes were steeper on absent trials than on present trials. There was no reliable effect of target orientation $[F(1,11)=2.21, p>.16]$, and there was no interaction between orientation and target presence $[F(1,11)=$ $2.34, p>.15]$.

\section{Experiment 1C: Left and Right Sides}

The results of Experiment 1C are shown in Figure 5B for displays composed of the left side (left panel) and the right side (right panel) of the conjoint shapes.

Left side. The left panel of Figure 5B shows that there was an orientation asymmetry, at absent and present trials, which went in the opposite direction to the one found with the complete shapes: A "vertical" target (i.e., the left side taken from the vertical shape) was detected faster than was an "oblique" target (i.e., the left side taken from the oblique shape). Furthermore, RTs were generally unaffected by set size, with the exception of those for an oblique target at absent trials. The search slopes confirmed this: The highest slope occurred for oblique absent displays, and the slopes for the other conditions were small.

An ANOVA on the RTs showed a reliable effect of target orientation $\left[F(1,9)=20.02, M S_{\mathrm{e}}=189,075, p<\right.$ $.002]$ : Search for a vertical target was faster than for an oblique target ( 882 vs. $1,237 \mathrm{msec}$ ). There was also a main effect of target presence $\left[F(1,9)=19.54, M S_{\mathrm{e}}=\right.$ $60,817, p<.002]$. There was no reliable main effect of set size $(F<1)$. However, target orientation interacted with set size $\left[F(2,18)=5.90, M S_{\mathrm{e}}=12,604, p<.02\right]$, indicating that RTs increased with set size for oblique targets (with an average slope of $17 \mathrm{msec} / \mathrm{item}$ ), whereas RTs for vertical targets slightly decreased as a function of set size (with an average slope of $-11 \mathrm{msec} / \mathrm{item}$ ). A similar trend occurred for absent and present trials, which interacted with set size $\left[F(2,18)=6.59, M S_{\mathrm{e}}=4,833, p<\right.$ $.008]$. The other interactions were not reliable $\left(F_{\mathrm{S}}<1\right)$.

An analysis on the slopes showed reliable effects of target presence $\left[F(1,9)=8.71, M S_{\mathrm{e}}=406, p<.02\right]$ and target orientation $\left[F(1,9)=7.60, M S_{\mathrm{e}}=1,059, p<.03\right]$.
Slopes (Table 3) were steeper for absent trials than for present trials and were steeper for oblique targets than for vertical targets. There was no interaction between target presence and orientation $(F<1)$.

Right side. The right panel of Figure 5B shows the results for contours relative to the right of the principal axis of the conjoint shape. An asymmetry occurred as a function of target orientation, but now in the opposite direction to that in the left-side condition: Search for an oblique target was faster than for a vertical target, at present and absent trials. Search functions were nearly flat in all conditions, with slopes for present trials being within the range (0-10 msec/item) for independence of set size, and slopes for absent trials just passing this range. Thus, search for both target orientations was fast and efficient.

An ANOVA on the RTs revealed reliable effects of target orientation $\left[F(1,9)=5.99, M S_{\mathrm{e}}=91,991, p<.04\right]$ and target presence $\left[F(1,9)=11.27, M S_{\mathrm{e}}=85,569, p<.009\right]$. On average, an oblique target was detected faster than was a vertical target (1,004 vs. $1,139 \mathrm{msec})$, and RTs were faster on present trials than on absent trials (982 vs. $1,161 \mathrm{msec})$. There was no reliable main effect of set size $[F(2,18)=1.18, p>.31]$, and set size did not interact with the other variables [target orientation $\times$ set size and the highest order interaction, $F_{\mathrm{S}}<1$; set size $\times$ target presence, $F(2,18)=2.77, p>.08$ ]. Finally, target orientation interacted with target presence $\left[F(1,9)=5.50, M S_{\mathrm{e}}=\right.$ $13,794, p<.05$ ], indicating that the RT difference between absent and present trials was higher for oblique targets than for vertical targets.

An ANOVA on the the slopes revealed a marginally reliable effect of target presence $\left[F(1,9)=4.97, M S_{\mathrm{e}}=\right.$ $293, p<.053$ ], with slopes being steeper on absent trials (other effects, $F \mathrm{~s}<1$ ).

\section{Discussion}

Experiment 1 showed three main results. First, search for the global orientation of the conjoint shape at small deviations from the vertical showed the same asymmetrical pattern as for the local orientation of line segments. This result replicates the one observed by Found and Müller (1997, Experiment 6). The shapes in their study and in the present experiment both contained an implicit axis of symmetry aligned with an axis of elongation. The finding of an orientation asymmetry in both cases supports the view that the detection of the global orientation was based on extraction of the principal axis. However, search performance was not identical. Found and Müller observed that search performance (in terms of RTs and search slopes) was basically the same for both lines and diamonds and that, at present trials, search slopes for diamonds were sufficiently shallow (below $6 \mathrm{msec} / \mathrm{item}$ ) to suggest that search was efficient. In contrast, search for the orientation of the conjoint shapes in our Experiment $1 \mathrm{~A}$ was impeded relative to the search for lines: RTs were elevated, and the steep slopes indicated inefficient search. It should be noted that, for present trials in Experiment $1 \mathrm{~A}$, the effects of RTs and slopes seemed to 
go in opposite directions: Although oblique targets generated faster RTs, search slopes were steeper for oblique targets than for vertical targets (Table 3 ). The steeper slope for oblique targets suggests that they might have produced a weaker signal than did the vertical targets (see also General Discussion).

What may account for the difference in search performance between the present results and Found and Müller's (1997) results? One possible factor is that the lines and diamonds in Found and Müller's displays were presented in a semi-ordered fashion, with roughly equal spacing between elements (Figure 2B). This may have generally facilitated performance (cf. Humphreys, Quinlan, \& Riddoch, 1989). In our displays, the elements were presented randomly at 16 possible locations; since the maximum set size used (10) was smaller, not all possible locations were occupied in any display, thereby making displays vary relatively more in terms of density and the spacing between elements. Our experiment showed that search for global orientation was slower and less efficient than search for local orientation.

Second, the search asymmetry for global orientation disappeared when the designated principal axis of the conjoint shape was presented at small deviations from the horizontal (Experiment 1B). We hypothesized that this effect would occur if search for the target orientation was based on the detection of the axis of symmetry in each shape, since horizontal symmetry is perceptually less salient than vertical symmetry. The results were consistent with this proposal. Apparently, the global orientation of the conjoint shape was determined by its principal axis of symmetry, which was less salient when this axis was horizontal. This result also suggests that elongation did not compensate for the reduced salience of the symmetry axis at the horizontal orientation. In Experiment 2, we examined the relative contributions of symmetry and elongation in further detail. Note that it is possible that the participants judged the horizontal shape according to the orientation of its designated "minor" axis (i.e., the one orthogonal to the principal axis), which was now vertically oriented. This would imply that the participants judged the orientation of a shape by whatever axis aligned with the environmental upright. However, it does not a priori exclude the influence of perceptual salience of internal shape axes on orientation judgments, since the perceptual salience of the axis that aligned with the vertical affected the orientation asymmetry. The observation that RTs were much slower when the axis was horizontal than when the axis was vertical supports the view that the detection of global orientation on the basis of axis information was more difficult in the former condition.

Third, the search asymmetry found with conjoint shapes in vertical and oblique orientations was not due to the processing of local contour information on either side of the principal axis. This was demonstrated, in Experiment $1 \mathrm{C}$, by the opposite search asymmetries for individual contours to the left and the right of the shape's principal axis. When the contours were presented jointly to compose the whole shape, the effects of separate contours to the left and the right of the principal axis canceled out, and the analysis of the global orientation seemed to operate on the global contour. In other words, processing of the local orientation of parts of the shape was qualitatively different from that of the global orientation of the shape as a whole.

Taken together, these results suggest that the detection of global orientation is determined by the principal axis of the shape, provided this axis is perceptually salient. Thus, the orientation of the conjoint shape in Experiment $1 \mathrm{~A}$ was determined by the orientation of its principal axis of symmetry and elongation; the resulting orientation asymmetry in the detection of vertical and oblique targets seemed to be based on the extraction of this axis. When the principal axis of the conjoint shape was oriented horizontally, the absence of an orientation asymmetry indicates that, in this condition, the orientation of the axis was not a salient cue to the global orientation. In Experiment 2 , we separately assessed the contributions of symmetry and elongation to search for global orientation.

\section{EXPERIMENT 2}

In Experiment 2, the observation of a search asymmetry as a function of the orientation of the principal axis of the conjoint shape (Experiment 1) was further explored with polygons, in which symmetry and elongation were systematically varied (Quinlan \& Humphreys, 1993). If global shape orientation was determined on the basis of the principal axis, then manipulating the perceptual salience of this axis should affect the orientation asymmetry. Under this assumption, the search asymmetry should disappear if the shape contains no axis of elongation or symmetry and thus has no salient axis-based description (Quinlan \& Humphreys, 1993). In contrast, if search was based on the analysis of the orientation of local contours, some search asymmetry might show up in one way or another (cf. Experiment 1C). We tested this hypothesis in Experiment 2A, by presenting displays composed of asymmetrical polygons containing no salient axis of elongation or symmetry (Figure 6, upper panel).

In Experiments 2B and 2C, orientation search was further tested for symmetrical and elongated polygons possessing, respectively, an axis of symmetry (but no axis of elongation) and an axis of elongation (but no axis of symmetry; Figure 6, middle and lower panels). The polygons were presented at orientations in which the designated axis of symmetry or elongation was vertical or oblique. In Experiment $1 \mathrm{~A}$, axes of elongation and symmetry were aligned within the conjoint shape and thus were confounded (cf. Found \& Müller, 1997). The present conditions allowed us to examine the relative contributions of symmetry and elongation to the orientation asymmetry.

Forty-eight students participated in the three experiments: asymmetrical (Experiment 2A), symmetrical (Experiment 2B), and elongated (Experiment 2C). Sixteen students were randomly assigned to each experiment. 
ASYMMETRICAL

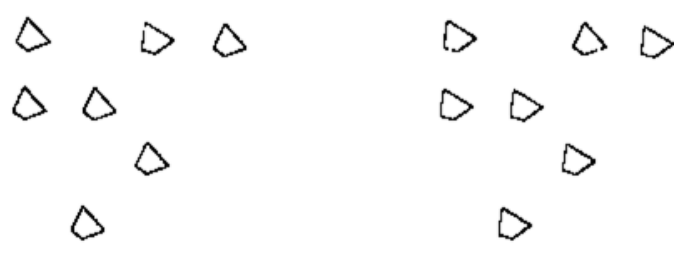

SYMMETRICAL

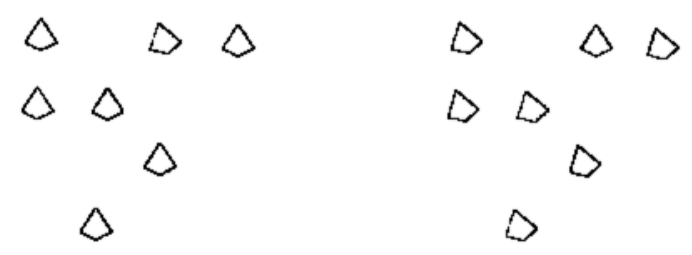

ELONGATED

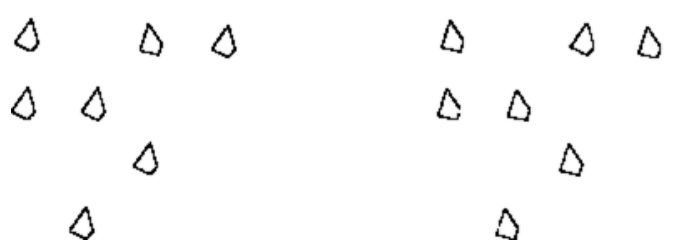

Figure 6. Example displays used in Experiment 2. The left displays show an oblique target among vertical distractors; the right displays show a vertical target among oblique distractors.

Results for the three conditions are presented in Figure 7; error rates are shown in Table 2, and search slopes are presented in Table 4.

\section{Experiment 2A: Asymmetrical}

Inspection of the results in the left panel of Figure 7 shows that there was no orientation asymmetry. At present trials, RTs were the same for vertical and oblique targets, specifically with 7- and 10-item displays; at absent trials, search for an oblique target was only a little faster than for a vertical target. This pattern of results is very similar to the one observed with horizontal and oblique conjoint shapes (Experiment 1B). With the exception of search for vertical targets at present trials, search functions increased with set size.

RTs. Tests of these observations by an ANOVA revealed a reliable main effect of set size $[F(2,30)=16.52$, $\left.M S_{\mathrm{e}}=38,683, p<.0001\right]$ and target presence $[F(1,15)=$ $\left.53.28, M S_{\mathrm{e}}=104,367, p<.0001\right]$. Most important, there was no reliable effect of target orientation $(F<1)$ : On average, search for an oblique target was only $54 \mathrm{msec}$ faster than search for a vertical target $(1,343 \mathrm{msec} v \mathrm{vs}$. $1,397 \mathrm{msec})$. There was one reliable interaction, between set size and target presence $\left[F(2,30)=9.17, M S_{\mathrm{e}}=24,971\right.$, $p<.0008$ ], indicating that search slopes were steeper for absent trials than for present trials (see below). The other interactions were not reliable [target orientation $\times$ set size, $F(2,30)=1.15, p>.32$; target orientation $\times$ target presence, $F<1$; the highest order interaction, $F(2,30)=1.00$, $p>$.37].

Slopes. An ANOVA showed a reliable effect of target presence $\left[F(1,15)=8.14, M S_{\mathrm{e}}=2,432, p<.02\right]$ : At each target orientation, absent slopes were steeper than present slopes (Table 4$)$. There was no effect of orientation $(F<$ $1)$, and there was no interaction $[F(1,15)=1.10, p>.30]$.

\section{Experiment 2B: Symmetrical}

The results (Figure 7, right panel) show an orientation asymmetry, with faster RTs to oblique targets than to vertical targets. RTs increased with set size in all conditions. The steep search slopes indicated inefficient search. This general pattern is very similar to the one observed for the conjoint shape (Experiment 1A).

RTs. There was a reliable effect of target orientation $\left[F(1,15)=18.45, M S_{\mathrm{e}}=458,399, p<.0006\right]$ : On average, an oblique target was detected $420 \mathrm{msec}$ faster than a vertical target $(1,162$ vs. $1,582 \mathrm{msec})$. There were also reliable effects of set size $\left[F(2,30)=24.63, M S_{\mathrm{e}}=46,946\right.$, $p<.0001]$ and target presence $\left[F(1,15)=20.30, M S_{\mathrm{e}}=\right.$ $211,448, p<.0005]$. Finally, target presence interacted with target orientation $\left[F(1,15)=6.93, M S_{\mathrm{e}}=26,021\right.$, $p<.02]$ and with set size $\left[F(2,30)=7.10, M S_{\mathrm{e}}=16,368\right.$, $p<.003]$. The former interaction indicated that the difference in RTs between absent and present trials was larger for vertical targets than for oblique targets; the latter interaction indicated that search slopes were steeper for absent trials than for present trials (Table 4). The other interactions were not reliable [target orientation $\times$ set size, $F(2,30)=2.99, p<.07$; the highest order interaction, $F<1$ ].

Slopes. Across target orientation, absent slopes were steeper than present slopes $\left[F(1,15)=24.18, M S_{\mathrm{e}}=463\right.$, $p<.001]$. There was no reliable effect of orientation $[F(1,15)=3.69, p>.07]$, and there was no interaction $(F<1)$.

Between-experiment comparisons. In order to compare the search asymmetry for the symmetrical shape with that found for the conjoint shape (Experiment 1A), an ANOVA was performed with experiment as a betweenparticipants factor. There was no reliable effect of experiment $[F(1,30)=1.99, p>.16]$, and experiment did not interact with target orientation $[F(1,30)=2.86, p>$ $.10]$. Thus, the search asymmetries found with the symmetrical shape and the conjoint shape were equivalent. In contrast, a comparison of the results for the symmetrical condition with those for the asymmetrical condition (Experiment 2A) revealed a reliable interaction between experiment and target orientation $\left[F(1,30)=7.17, M S_{\mathrm{e}}=\right.$ 

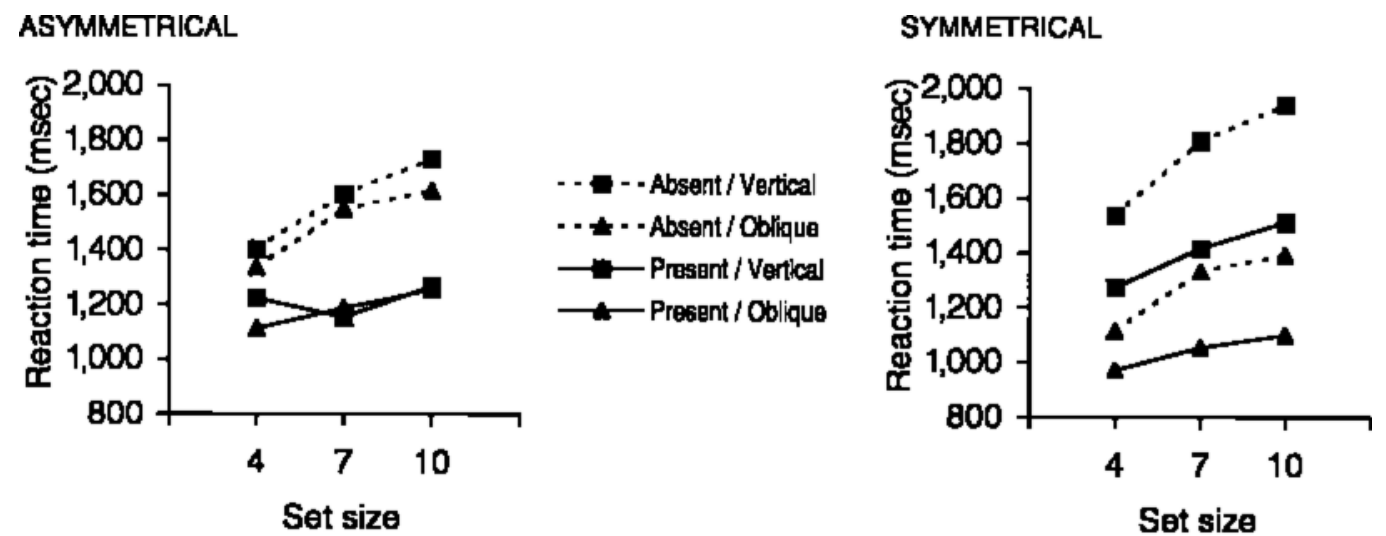

ELONGATED

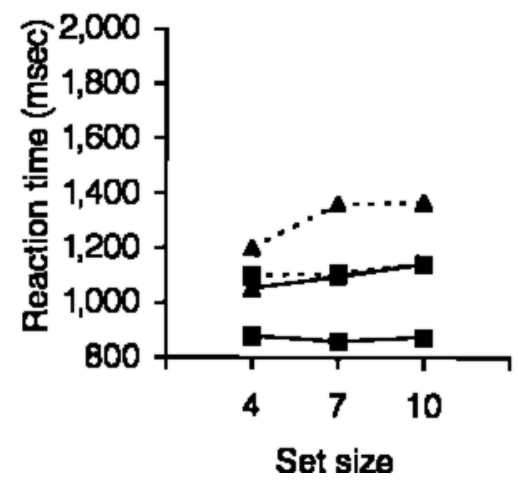

Figure 7. Mean RTs per condition in Experiment 2. The labels indicate target presence and target orientation.

$927,380, p<.02]$, indicating that search was faster for oblique targets than for vertical targets for the symmetrical condition but not for the asymmetrical condition. There was no main effect of experiment $(F<1)$, indicating that overall RTs to displays of symmetrical and asymmetrical shapes were similar.

\section{Experiment 2C: Elongated}

The lower panel of Figure 7 shows the results for the elongated condition. An orientation symmetry occurred in the opposite direction to that in the symmetrical condition: Search was faster for a vertical target than for an oblique target. This result is surprising, since it suggests that orientation detection was not based on the principal axis of elongation. In fact, this result is very similar to the result found for the left-side condition in Experiment 1C, in which the same asymmetry was observed for the contours to the left of the principal axis of the conjoint shape. We will return to this issue in the Discussion section. Search slopes in the elongated condition were shallow, with the exception of RTs for an oblique target on absent trials. Search for a vertical target was unaffected by the number of distractors, whereas search for an oblique target slowed down as a function of set size (see below).

RTs. An ANOVA showed a reliable main effect of target orientation $\left[F(1,15)=8.72, M S_{\mathrm{e}}=239,592, p<.01\right]$ :
A vertical target was detected faster than was an oblique target ( 993 vs. $1,202 \mathrm{msec}$ ). There were also reliable main effects of set size $\left[F(2,30)=6.99, M S_{\mathrm{e}}=13,063, p<\right.$ $.004]$ and target presence $\left[F(1,15)=31.64, M S_{\mathrm{e}}=\right.$ $79,156, p<.0001]$. Target orientation interacted with set size $\left[F(2,30)=8.54, M S_{\mathrm{e}}=7,068, p<.002\right]$, indicating that search slopes were steeper for oblique targets than for vertical targets. The other interactions were not reliable [target orientation $\times$ target presence and the highest order interaction, $F_{\mathrm{S}}<1$; set size $\times$ target presence, $F(2,30)=$ $2.14, p>.13]$.

Slopes. An ANOVA showed a reliable effect of target orientation $\left[F(1,15)=14.20, M S_{\mathrm{e}}=357, p<.002\right]$ : Search slopes were steeper for an oblique target than for a vertical target (Table 4$)$. There was no reliable effect of target presence $[F(1,15)=1.53, p>.23]$, and there was no interaction $(F<1)$.

\section{Discussion}

Experiment 2 was designed as a further test of the proposal that the perception of the global orientation of 2-D shapes depends on the detection of the orientation of a salient principal axis of symmetry and/or elongation, computed on the basis of global contour information. We reasoned that if global orientation was based on the principal axis (cf. Experiment 1), then the orientation asym- 
metry for vertical and oblique orientations should be affected when the salience of this axis, in these orientations, was reduced. The results of the asymmetrical condition (Experiment 2A) provided support for this proposal, since the orientation asymmetry disappeared when the shapes contained no axis of symmetry or elongation. This does not automatically imply, however, that orientation search for the asymmetrical shape was based on the distinctive orientation of some local contour (cf. Experiment 1C). Rather, the absence of any search asymmetry indicates that orientation search was based on an analysis of the global contour instead of on local contours. The absence of an asymmetry indicates that the designated principal axis was not used to describe the orientation of the global contour. Thus, the possibility remains that some other, perhaps oblique, axis was used to describe the orientation of the asymmetrical shape. For example, one could describe the orientation axis of the asymmetrical shape as bisecting opposing edges (e.g., running from below left to upper right through the vertical shape; cf. the oblique axis drawn in the asymmetrical shape in Figure 3), rather than opposing vertices or angles. This axis is oblique in both target orientations, rotated between approximately $32^{\circ}$ and $50^{\circ}$ from the vertical; since search asymmetries do not occur between oblique orientations (around $45^{\circ}$ from the vertical; see Marendaz et al., 1991), the absence of an asymmetry is consistent with the choice of an alternative axis. ${ }^{2}$

The symmetrical and elongated conditions were run to separately assess the contribution of axes of elongation and symmetry to the orientation asymmetry. These conditions yielded contrasting results. First, the same orientation asymmetry was observed for symmetrical shapes (Experiment 2B) as for conjoint shapes (Experiment 1A): RTs did not differ between both types of displays. Since the symmetrical shapes were not elongated, this result shows that elongation was not an important determinant of the orientation asymmetry with this shape; rather, the axis of symmetry seemed to be the major (and sufficient) determinant of global orientation.

Second, search for the orientation of elongated shapes yielded the opposite orientation asymmetry to that for the symmetrical shapes: Search was faster for a target whose axis of elongation was vertical rather than oblique. This result is surprising, since it suggests not only that elongation did not contribute to the orientation asymmetry for the conjoint shape in Experiment 1 but also that it would have a qualitatively different effect on the search asymmetry. Therefore, it seems unlikely that the designated axis of elongation in this shape was used as a cue to its global orientation. Two alternatives remain to account for this result. The first is that the global orientation of the elongated shape was described along a different axis (cf. the asymmetrical condition). Under this assumption, the orientation axis should be more vertical in the oblique target orientation and should be more oblique in the vertical target orientation in order to produce the orientation asymmetry. One candidate axis would be the one bisecting opposing contours from below right to upper left (see the oblique axis drawn in the elongated shape in Figure 3). However, it is not clear why this axis would be more salient than any other axis in another orientation. The second alternative is that search for the target was guided by the orientation of a distinctive local contour. In order for a salient feature to produce the observed search asymmetry in the present condition, the orientation of the contour should be (close to) vertical in the oblique target condition and should be more oblique in the vertical target condition. In fact, a similar effect was observed in the left-side condition in Experiment $1 \mathrm{C}$, in which the individual contours to the left of the principal axis of the conjoint shape yielded an opposite orientation asymmetry to those yielded by the contours to the right. The observation that, in the elongated condition, search slopes were rather shallow, relative to those in the other conditions, supports this proposal. However, further manipulations would be needed to examine which local contour would account for the orientation asymmetry.

Whatever the nature of the orientation asymmetry for the elongated shape, the results show that the designated axis of elongation did not seem to be used in the detection of the global orientation. This observation is compatible with results obtained by Quinlan and Humphreys (1993), who studied, with the same polygons, the effect of axes of symmetry and elongation on shape perception. In a rating study, these authors observed that the axis of elongation of the elongated shape was not chosen as a salient principal axis; increasing the aspect ratio (maximum 2) to enhance elongation did not change this result. Moreover, matching elongated shapes at different orientations was not facilitated by explicitly presenting the axis of elongation; in contrast, providing the axis of symmetry did facilitate matching performance for symmetrical and conjoint shapes. Nevertheless, as we will demonstrate in Experiment 3, shape elongation can, under some conditions, determine the perception of global orientation.

In sum, the results of Experiments 1 and 2 provide evidence that search for the global orientation of the polygons depends on the detection of a salient principal axis, which is extracted at least on the basis of contour symmetry. Orientation asymmetries were observed for shapes containing an axis of symmetry. On the other hand, the role of shape elongation in the determination of an orientation axis, at least for the elongated polygon used here, seemed to be less important.

\section{EXPERIMENT 3}

In Experiment 3, we extended the axis-based account of global orientation processing to shapes composed of curved contours rather than straight contours. The polygons used in Experiments 1 and 2 had straight contours with distinct local orientations. Although some of the results in these experiments suggested that it is unlikely that search for the target orientation was based on local contour orientations, this possibility cannot be completely discarded. For example, the search performance for the elongated shape in Experiment 2C, whose axis of 


\section{CONJOINT CURVE}

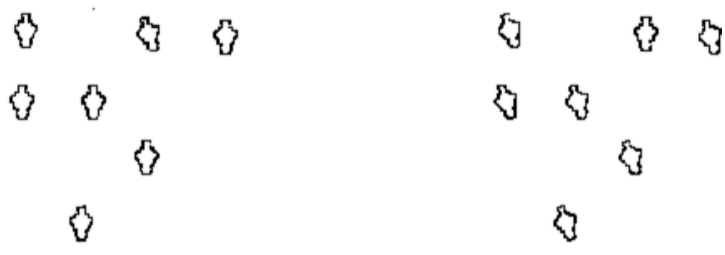

ELONGATED CURVE

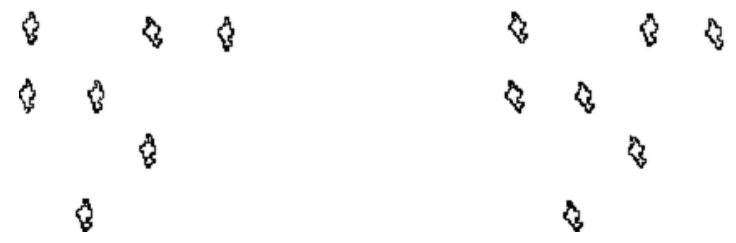

Figure 8. Example displays used in Experiment 3. The left displays show an oblique target among vertical distractors; the right displays show a vertical target among oblique distractors.

elongation apparently was not salient (cf. Quinlan \& Humphreys, 1993), suggests that the detection of the target orientation was dependent on local rather than global contour information. By presenting shapes with curved contours, the possible influence of distinctive local contour orientations on search for the target orientation can be reduced. Displays of two different shapes were presented (Figure 8). In Experiment 3A, a conjoint curve was used, containing aligned axes of elongation and symmetry. In Experiment 3B, an elongated curve was presented, containing an implicit axis of elongation but no axis of symmetry. The two shapes thus were structurally equivalent to the conjoint and elongated polygons, respectively. Search performance for the orientation of these shapes was tested in the same manner as in Experiments 1 and 2. The observation of orientation search asymmetries with these shapes would further support the role of principal axis information in the detection of global orientation. Moreover, the observation of an orientation asymmetry for the elongated curve would provide evidence that the perception of global orientation can be based on an axis of elongation, when there is no competition from other local contours.

Thirty-two students participated Experiments $3 \mathrm{~A}$ and 3B; 16 students were randomly assigned to each experiment. Results are presented in Figure 9 (RTs), Table 2 (error rates), and Table 4 (search slopes).

\section{Experiment 3A: Conjoint Curve}

The left panel of Figure 9 shows the RTs for the conjoint curve as a function of target orientation, target pres- ence, and set size. These results show an orientation asymmetry: Search for an oblique target was faster than for a vertical target (1,019 vs. 1,557 msec). Slopes were steep, suggesting inefficient search for both target orientations (Table 4).

RTs. An ANOVA showed reliable effects of target orientation $\left[F(1,15)=42.00, M S_{\mathrm{e}}=331,168, p<.0001\right]$, set size $\left[F(2,30)=28.76, M S_{\mathrm{e}}=56,797, p<.0001\right]$, and target presence $\left[F(1,15)=18.70, M S_{\mathrm{e}}=203,607, p<\right.$ .0007]. Target orientation also interacted with set size $\left[F(2,30)=9.83, M S_{\mathrm{e}}=26,463, p<.0006\right]$, indicating that the search slopes were steeper for vertical than for oblique targets. Finally, set size interacted with target presence $\left[F(2,30)=8.35, M S_{\mathrm{e}}=17,334, p<.002\right]$, showing that search slopes were steeper for absent than for present trials. The remaining interactions were not reliable [target orientation $\times$ target presence, $F(1,15)=1.81, p>$ .19 ; the highest order interaction, $F(2,30)=1.63, p>.21]$.

Slopes. Analysis of the slopes showed reliable effects of target presence $\left[F(1,15)=9.42, M S_{\mathrm{e}}=1,699, p<\right.$ $.008]$ and target orientation $\left[F(1,15)=11.85, M S_{\mathrm{e}}=\right.$ $2,256, p<.004]$. Slopes were steeper for absent trials and for vertical targets. There was no reliable interaction between orientation and target presence $[F(1,15)=2.49$, $p>.13]$.

Between-experiment comparison. A comparison of search times with those for the conjoint polygons (Experiment 1A) through an ANOVA (with experiment as a between-participants factor) yielded no reliable effect of experiment $(F<1)$ : RTs were not reliably faster with the conjoint polygon than with the conjoint curve $(1,188$ vs. $1,305 \mathrm{msec})$. However, experiment interacted with target orientation $\left[F(1,30)=7.48, M S_{\mathrm{e}}=397,645, p<.02\right]$ : This shows that the orientation asymmetry in terms of absolute differences in RTs was larger for the conjoint curve than for the conjoint polygon (538 vs. $199 \mathrm{msec}$ ). Experiment also interacted with target orientation and set size $\left[F(2,60)=5.86, M S_{\mathrm{e}}=26,176, p<.005\right]$. This indicates that search slopes were steeper for a vertical conjoint curve than for a vertical conjoint polygon: For example, at present trials, the slope for a vertical target was $52.3 \mathrm{msec} /$ item for the conjoint curve but was only $17.9 \mathrm{msec} / \mathrm{item}$ for the conjoint polygon. In contrast, slopes for oblique targets were more similar between the two experiments (e.g., at present trials, 28.2 and $19.8 \mathrm{msec}$ / item for conjoint polygons and conjoint curves, respectively). Thus, search for a vertical target was less efficient for the conjoint curve than for the conjoint polygon, whereas search for an oblique target was equally efficient (though, in both cases, affected by set size).

\section{Experiment 3B: Elongated Curve}

The results for the elongated curve (see Figure 9, right panel) show an orientation asymmetry, with faster RTs for an oblique target than for a vertical target (1,164 vs. $1,829 \mathrm{msec})$. This orientation asymmetry was very similar to the one with the conjoint curve, although the elongated curve had no axis of symmetry: Apparently, orien- 
CONJOINT CURVE

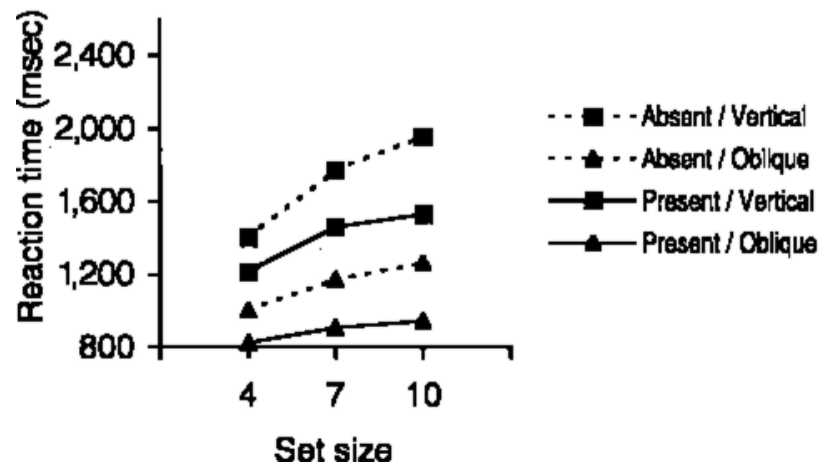

ELONGATED CURVE

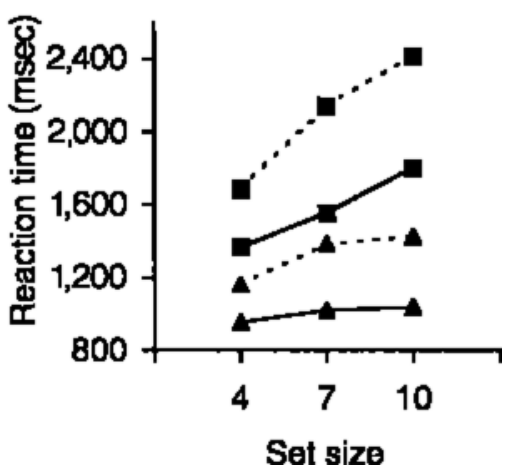

Figure 9. Mean RTs per condition in Experiment 3. The labels indicate target presence and target orientation.

tation search was based on the axis of elongation. Search slopes were steep for all conditions and were similar to the ones with the conjoint curve. Also, search slopes were steeper for vertical targets than for oblique targets (Table 4).

RTs. There were reliable main effects of target orientation $\left[F(1,15)=86.69, M S_{\mathrm{e}}=245,101, p<.0001\right]$, set size $\left[F(2,30)=38.79, M S_{\mathrm{e}}=60,084, p<.0001\right]$, and target presence $\left[F(1,15)=55.11, M S_{\mathrm{e}}=148,615, p<.0001\right]$. Target orientation interacted with set size $[F(2,30)=$ $\left.15.79, M S_{\mathrm{e}}=42,681, p<.0001\right]$ and with target presence $\left[F(1,15)=10.00, M S_{\mathrm{e}}=41,715, p<.007\right]$. These interactions indicate that search slopes were steeper and the difference in RTs between absent and present trials was larger for vertical targets than for oblique targets (RT differences, 506 and $319 \mathrm{msec}$ for vertical and oblique targets, respectively). Set size also interacted with target presence $\left[F(2,30)=15.72, M S_{\mathrm{e}}=17,273, p<.0001\right]$, indicating that slopes were steeper for absent trials than for present trials. The highest order interaction was not reliable $[F(2,30)=1.20, p>.31]$.

Slopes. There were reliable effects of target presence $\left[F(1,15)=17.92, M S_{\mathrm{e}}=1,391, p<.0008\right]$ and target orientation $\left[F(1,15)=25.43, M S_{\mathrm{e}}=2,927, p<.0002\right]$, confirming that search slopes were steeper for absent trials and for vertical targets. There was no reliable interaction $[F(1,15)=1.92, p>.18]$.

Between-experiment comparison. Search for the elongated curve was compared with that for the conjoint curve through an ANOVA with experiment as a betweenparticipants factor. Comparison of the results in Figure 9 suggests that RTs were generally faster for the conjoint curve than for the elongated curve. This main effect of experiment was only marginally reliable $[F(1,30)=3.39$, $\left.M S_{\mathrm{e}}=1,254,927, p=.07\right]$ : On average, RTs to the elongated curve were $211 \mathrm{msec}$ slower than those to the conjoint curve (1,516 vs. 1,305 msec). Experiment did not interact with target orientation $(F<1)$, indicating the equivalence of the orientation asymmetries in both experiments. However, experiment interacted with target ori- entation and target presence $\left[F(1,30)=7.61, M S_{\mathrm{e}}=\right.$ $31,572, p<.01]$. This interaction was due to the fact that, at absent trials, the difference between RTs for vertical and oblique targets was larger for the elongated curve $(817 \mathrm{msec})$ than for the conjoint curve $(583 \mathrm{msec})$. Experiment also marginally interacted with set size and target presence $\left[F(2,60)=2.83, M S_{\mathrm{e}}=19,276, p=.06\right]$. This interaction was probably due to the steeper search slope at absent vertical trials for the elongated curve relative to the conjoint curve (121.4 vs. $92.3 \mathrm{msec} / \mathrm{item})$.

\section{Discussion}

The results of Experiment 3 demonstrate that the orientation asymmetry was not confined to shapes with straight contours possessing distinctive local orientations but also occurred for shapes with curved contours with less salient local orientations but possessing a salient principal axis. This provides further evidence that the orientation asymmetry was based on the orientation of the principal axis of the shape, and not merely on the basis of the orientation of local contours. Search performance with conjoint curves (Experiment $3 \mathrm{~A}$ ) was equivalent to that with conjoint polygons in terms of overall RTs, although the orientation asymmetry was larger with the conjoint curves. The orientation asymmetry also occurred with elongated curves (Experiment 3B), a result that contradicts the one observed for elongated polygons (Experiment 2C). Apparently, the designated axis of elongation of the elongated curve was used to extract global orientation, and this axis was more salient than that of the elongated polygon. This suggests that the orientation of an axis of elongation (in the absence of symmetry) can determine the perception of global orientation in the same way as an axis of symmetry. ${ }^{3}$ A comparison between performance for the conjoint and elongated curves revealed that symmetry had a general effect on search for global orientation, since it led to faster RTs to conjoint curves than to elongated curves. Shape symmetry thus facilitated the selection of the principal axis of the conjoint curve in order to determine the axial orientation. 


\section{GENERAL DISCUSSION}

The present experiments demonstrate that the detection of the global orientation of 2-D shapes can be accomplished by the selection of a salient axis on the basis of contour information. The orientation of this axis determines the perceived orientation of the shape. This conclusion is based on the observation of search asymmetries as a function of the orientation of the designated principal axis within the shapes, analogous to search asymmetries for the local orientation of contours. Experiments 1-3 showed search asymmetries for the global orientation of 2-D shapes, provided they possess a salient principal axis of symmetry or elongation. These experiments also yielded information about the conditions under which axis-based orientation asymmetries can occur, or, in other words, when an implicit axis is "salient" to provide a cue for the selection of shape orientation. Experiment 1 showed asymmetries for a shape with a joint axis of symmetry and elongation in small deviations from the vertical, not the horizontal. Experiment 2 showed orientation asymmetries as a function of an axis of symmetry, but not elongation. Experiment 3 showed axisbased orientation asymmetries for shapes with curved contours in which the possible influence of competing local orientations was reduced. With these shapes, orientation asymmetries occurred as a function of axes of both symmetry and elongation. We suggest that the contrasting results for the elongated shapes (Experiments 2C and $3 \mathrm{~B}$ ) can be attributed to the reduced salience of the axis of elongation of the elongated polygon. This reduced salience was probably due to the presence of competing local contour orientations in the polygon.

In general, across the experiments, detection of global shape orientation was rather slow and inefficient, as evidenced by steep search slopes. In contrast, search for the local orientation of conjunctions of contours (Experiment 1C) was fast and efficient. This contrasts with some of the findings by Found and Müller (1997, Experiment 6), who observed effortless search for both local and global orientation. However, these authors also observed conditions in which search for global orientation was not efficient. For example, search functions for a tilted diamond among heterogeneous distractors were steep, whereas slopes for tilted lines among distractors in mixed orientations remained shallow (Found \& Müller, 1997, Experiment 1).

Several factors may determine inefficient search for global orientation, including distinctive processing dynamics (serial vs. parallel), target discriminability and sensory factors, and eye movements. To the extent that set-size effects (i.e., steep RT slopes) reflect a different processing mechanism, the search functions raise the possibility that search for global shape orientation proceeds in a serial manner (i.e., requires selective attention). For example, the selection of an orientation axis on the basis of shape symmetry may require the integration of local contour orientations, and search then involves de- tecting a symmetrical conjunction of local contours for each item, which may proceed in a serial, attentional manner. At first sight, this seems to contradict results from studies on symmetry detection showing that symmetry is detected without attention (e.g., Baylis \& Driver, 1994, 1995; Driver, Baylis, \& Rafal, 1992; Wagemans et al., 1992). However, these studies tested the detection of symmetry within single patterns. For example, Baylis and Driver (1994) observed that symmetry detection was unaffected by the number of discontinuities in shape contour, whereas the detection of repetition of such discontinuities was a function of the number of discontinuities. In order for symmetry to guide selection of target shapes, however, it should be detectable without effort in displays containing multiple items. Recently, this issue was examined by Olivers and van der Helm (1998), who observed that search for a symmetrical target pattern (e.g., dot patterns, polygons, or solid surfaces) among asymmetrical distractors was attentional (serial), as was search for an asymmetrical target among symmetrical distractors. Moreover, there was no search asymmetry in the detection of symmetrical versus asymmetrical targets. Thus, according to these results, symmetry is not a basic (preattentive) visual feature. Olivers and van der Helm's findings are compatible with the results of our study, in which effects of symmetry were tested indirectly through judgments of orientation. Axes of symmetry facilitated orientation processing in a general way (cf. Experiment 3), although search remained attentional. As Baylis and Driver $(1994,1995)$ argued, the general advantage for symmetry detection could be a by-product of preattentive figure-ground segmentation and part decomposition processes: Following an initial segmentation of a visual scene, the description of objects into parts would be easier for symmetrical objects, since boundaries on both sides of the symmetry axis have the same part description (i.e., concave and convex regions match exactly across the axis of symmetry). Although our study did not test judgments of symmetry, it is likely that symmetry detection following figure-ground segmentation and grouping processes was involved in the computation of an axis for the shapes.

Alternatively, the difference in search efficiency for local and global orientation may be attributed to signalto-noise ratios or discriminability between targets and distractors. For example, it is likely that the global orientation has a weaker signal than local orientation, due to competition from local contours (cf. the polygons in Experiments 1 and 2). Some evidence for this comes from a comparison between search slopes for conjunctions of local contours (Experiment 1C) and shapes with salient principal axes (Experiments 1A, 2B, and 3). In the latter conditions, search slopes were considerably steeper than in the former. Finally, eye movements could have influenced search performance, although it is unlikely that they would have affected the search asymmetries per se (e.g., Carrasco, Evert, Chang, \& Katz, 1995; Carrasco et al., 1998; Klein \& Farrell, 1989). Given that displays were 
viewed until response, eye movements could have allowed foveal processing of individual elements or regions of the display, resulting in steeper search slopes.

The argument of signal-to-noise ratio may also apply to the role of elongation in shape orientation. Intuitively, it seems obvious that shape elongation can be a cue to global orientation. However, this effect clearly depends on perceptual salience, as a comparison between the results for elongated curves and polygons showed. The presence of competing local orientations may have prevented the participants from selecting the principal axis of the elongated polygon as a cue to global orientation. On the other hand, aspect ratio can enhance the salience of shape elongation (Sekuler, 1996). Detecting a salient axis of elongation requires the computation of the ratio between the width and the length of a shape. This process could operate on visual information at a different spatial scales (Burbeck \& Pizer, 1995; Burbeck \& Zauberman, 1997). Also, there is some psychophysical and neurophysiological evidence that the visual system computes implicit or "medial" axes running through the center of shapes (Kovács \& Julesz, 1994; Lee, Mumford, \& Schiller, 1995). However, at present, it is unclear whether the detection of axes of elongation in multi-item displays (even on the basis of coarse "blob-like" information) can occur without selective attention. Future research is needed to unravel this issue.

The present results shed further light on the relation between local and global orientation and extend the basic findings by Found and Müller (1997) by emphasizing the role of overall shape geometry (symmetry and elongation) in perceived shape orientation. It seems that the processes and representations underlying local and global orientation are linked. Detection of global orientation results from the processing of contour information (including local orientation information) in which grouping and segmentation processes operate on contours to generate a representation of global shape and orientation (cf. Found \& Müller, 1997). Detection of shape symmetry and elongation may take place at this stage. Thus, although axes of symmetry and elongation can be used to detect global orientation, they are derived properties emerging from an analysis of the global shape.

\section{REFERENCES}

Appelle, S. (1972). Perception and discrimination as a function of stimulus orientation: The oblique effect in man and animals. Psychological Bulletin, 78, 266-278.

BAYlis, G. C., \& Driver, J. (1994). Parallel computation of symmetry but not repetition within single visual shapes. Visual Cognition, 1, 377-400.

Baylis, G. C., \& Driver, J. (1995). Obligatory edge assignment in vision: The role of figure and part segmentation in symmetry detection. Journal of Experimental Psychology: Human Perception \& Performance, 21, 1323-1342.

BIEDERMAN, I. (1987). Recognition-by-components: A theory of human image understanding. Psychological Review, 94, 115-147.

Bilsky, A. B., \& Wolfe, J. M. (1995). Part-whole information is useful in visual search for size $\times$ size but not orientation $\times$ orientation conjunctions. Perception \& Psychophysics, 57, 749-760.
Blum, H., \& Nagel, R. N. (1978). Shape description using weighted symmetric axis features. Pattern Recognition, 10, 167-180.

Bravo, M., \& Blake, R. (1990). Preattentive vision and perceptual groups. Perception, 19, 515-522.

Burbeck, C. A., \& Pizer, S. M. (1995). Object representation by cores: Identifying and representing primitive spatial regions. Vision Research, 35, 1917-1930.

Burbeck, C. A., \& Zauberman, G. S. (1997). Across-object relationships in perceived object orientation. Vision Research, 37, 879-884.

Carrasco, M., Evert, D. L., Chang, I., \& Katz, S. M. (1995). The eccentricity effect: Target eccentricity affects performance on conjunction searches. Perception \& Psychophysics, 57, 1241-1261.

Carrasco, M., McLean, T. L., Katz, S. M., \& Frieder, K. S. (1998). Feature asymmetries in visual search: Effects of display duration, target eccentricity, orientation and spatial frequency. Vision Research, 38, 347-374.

Carrasco, M., \& Yeshurun, Y. (1998). The contribution of covert attention to the set-size and eccentricity effects in visual search. Journal of Experimental Psychology: Human Perception \& Performance, 24, 673-692.

Cavanagh, P., Arguin, M., \& Treisman, A. (1990). Effect of surface medium on visual search for orientation and size features. Journal of Experimental Psychology: Human Perception \& Performance, 16, 479-491.

Cheal, M. L., \& Lyon, D. R. (1992). Benefits from attention depend on the target type in location-precued discrimination. Acta Psychologica, 81, 243-267.

Chelazzi, L. (1999). Serial attention mechanisms in visual search: A critical look at the evidence. Psychological Research, 62, 195-219.

Corballis, M. C., \& Roldan, C. E. (1975). Detection of symmetry as a function of angular orientation. Journal of Experimental Psychology: Human Perception \& Performance, 1, 221-230.

DAVIS, G., \& DrIVER, J. (1994). Parallel detection of Kanisza subjective figures in the human visual system. Nature, 371, 791-793.

Driver, J., Baylis, G. C., \& Rafal, R. D. (1992). Preserved figureground segregation and symmetry perception in visual neglect. $\mathrm{Na}$ ture, 360, 73-75.

Duncan, J., \& Humphreys, G. W. (1989). Visual search and stimulus similarity. Psychological Review, 96, 433-458.

Enns, J. T., \& Rensink, R. A. (1990). Influence of scene-based properties on visual search. Science, 247, 721-723.

Enns, J. T., \& Rensink, R. A. (1991). Preattentive recovery of threedimensional orientation from line drawings. Psychological Review, 98, 335-351.

Foster, D. H., \& WARD, P. A. (1991a). Asymmetries in oriented-line detection indicate two orthogonal filters in early vision. Proceedings of the Royal Society of London: Series B, 243, 75-81.

Foster, D. H., \& WARD, P. A. (1991b). Horizontal-vertical filters in early vision predict anomalous line-orientation identification frequencies. Proceedings of the Royal Society of London: Series B, 243, 83-86.

Foster, D. H., \& Westland, S. (1995). Orientation contrast vs. orientation in line-target detection. Vision Research, 35, 733-738.

Found, A., \& MÜlLER, H. J. (1997). Local and global orientation in visual search. Perception \& Psychophysics, 59, 941-963.

Grossberg, S., Mingolla, E., \& Ross, W. D. (1994). A neural theory of attentive visual search: Interactions of boundary, surface, spatial, and object representations. Psychological Review, 101, 470-489.

Heeley, D. W., Buchanan-Smith, H. M., Cromwell, J. A., \& Wright, J. S. (1997). The oblique effect in orientation acuity. Vision Research, 37, 235-242.

HowARD, I. P. (1982). Human visual orientation. New York: Wiley. Humphreys, G. W. (1983). Reference frames and shape perception. Cognitive Psychology, 15, 151-196.

Humphreys, G. W. (1984). Shape constancy: The effects of changing shape orientation and the effects of changing the position of focal features. Perception \& Psychophysics, 36, 50-64.

Humphreys, G. W., \& Quinlan, P. T. (1988). Priming effects between two-dimensional shapes. Journal of Experimental Psychology: Human Perception \& Performance, 14, 203-220.

Humphreys, G. W., Quinlan, P. T., \& RidDOCH, M. J. (1989). Group- 
ing processes in visual search: Effects with single- and combinedfeature targets. Journal of Experimental Psychology: General, 118, 258-279.

Iтті, L., \& Kосн, C. (2000). A saliency-based search mechanism for overt and covert shifts of visual attention. Vision Research, 40, 14891506.

JEANNEROD, M. (1997). The cognitive neuroscience of action. Oxford: Blackwell.

KANADE, T. (1981). Recovery of the three-dimensional shape of an object from a single view. Artificial Intelligence, 17, 409-460.

Klein, R, \& Farrell, M. (1989). Search performance without eye movements. Perception \& Psychophysics, 46, 476-482.

Kovács, I., \& Julesz, B. (1994). Perceptual sensitivity maps within globally defined visual shapes. Nature, 370, 644-646.

LAuwereyns, J., \& D'YDEWAlle, G. (1997). Global orientation disrupts the detection of a similar local orientation. Perception, 26, 1259-1270.

Lee, T. S., Mumford, D., \& Schiller, P. H. (1995). Neuronal correlates of boundary and medial axis representations in primate striate cortex [Abstract]. Investigative Ophthalmology\& Visual Science, $\mathbf{3 6}$, S477.

Li, W., \& Westheimer, G. (1997). Human discrimination of the implicit orientation of simple symmetrical patterns. Vision Research, 37, 565-572.

МАCH, E. (1959). The analysis of sensations and the relation of the physical to the psychical. New York: Dover. (Original work published 1886.)

Marendaz, C. (1998). Nature and dynamics of reference frames in visual search for orientation: Implications for early visual processing. Psychological Science, 9, 27-32.

Marendaz, C., Stivalet, P., Barraclough, L., \& Walkowiac, P. (1993). Effect of gravitational cues on visual search for orientation. Journal of Experimental Psychology: Human Perception \& Performance, 19, 1266-1277.

Marendaz, C., Stivalet, P., \& Genon, D. (1991). Coding of orientation in early vision: Search asymmetry revisited. Cahiers de Psychologie Cognitive/European Bulletin of Cognitive Psychology, 11, 427-440.

MARr, D. (1982). Vision. New York: Freeman.

MarR, D., \& Nishihara, H. K. (1978). Representation and recognition of the spatial organization of three-dimensional shapes. Proceedings of the Royal Society of London: Series B, 200, 269-294.

McElree, B., \& Carrasco, M. (1999). The temporal dynamics of visual search: Evidence for parallel processing in feature and conjunction searches. Journal of Experimental Psychology: Human Perception \& Performance, 25, 1517-1539.

Meigen, T., Lagreze, W.-D., \& Bach, M. (1994). Asymmetries in preattentive line detection. Vision Research, 34, 3103-3109.

NeIsSER, U. (1967). Cognitive psychology. New York: Appleton.

Nothdurft, H. C. (1991). Texture segmentation and pop-out from orientation contrast. Vision Research, 31, 1073-1078.

Olivers, C. N. L., \& VAN der Helm, P. A. (1998). Symmetry and selective attention: A dissociation between effortless perception and serial search. Perception \& Psychophysics, 60, 1101-1116.

Orban, G. A., Vandenbussche, E., \& Vogels, R. (1984). Human orientation discrimination tested with long stimuli. Vision Research, 24, 121-128.

PALMer, J. (1994). Set-size effects in visual search: The effect of attention is independent of the stimulus for simple tasks. Vision Research, 34, 1703-1721.

Palmer, J. (1995). Attention in visual search: Distinguishing four causes of a set-size effect. Current Directions in Psychological Science, 4, 118-123.

PALMer, S. E. (1980). What makes triangles point: Local and global effects in configurations of ambiguous triangles. Cognitive Psychology, 12, 285-305.

PALMer, S. E. (1985). The role of symmetry in shape perception. Acta Psychologica, 59, 67-90.

Palmer, S. E., \& Hemenway, K. (1978). Orientation and symmetry:
Effects of multiple, rotational, and near symmetries. Journal of Experimental Psychology: Human Perception \& Performance, 4, 691702.

Pashler, H. (1990). Coordinate frame for symmetry detection and object recognition. Journal of Experimental Psychology: Human Perception \& Performance, 16, 150-163.

Quinlan, P. T. (1991). Differing approaches to two-dimensional shape recognition. Psychological Bulletin, 109, 224-241.

Quinlan, P. T., \& Humphreys, G. W. (1993). Perceptual frames of reference and two-dimensional shape recognition: Further examination of internal axes. Perception, 22, 1343-1364.

SAARINEN, J. (1994). Visual search for global and local stimulus features. Perception, 23, 237-243.

Sekuler, A. B. (1996). Axis of elongation can determine reference frames for object perception. Canadian Journal of Experimental Psychology, 50, 270-278.

SHAW, M. L. (1984). Division of attention among spatial locations: A fundamental difference between detection of letters and detection of luminance increments. In H. Bouma \& D. G. Bouwhuis (Eds.), Attention and performance $X$ (pp. 109-120). Hillsdale, NJ: Erlbaum.

Sun, J. Y., \& Perona, P. (1996). Preattentive perception of elementary three-dimensional shapes. Vision Research, 36, 2515-2529.

TownsEND, J. T. (1972). Some results concerning the identifiability of parallel and serial processes. British Journal of Mathematical \& Statistical Psychology, 25, 168-199.

TownsEND, J. T. (1990). Serial versus parallel processing: Sometimes they look like Tweedledum and Tweedledee but they can (and should) be distinguished. Psychological Science, 1, 46-54.

Treisman, A. (1985). Preattentive processing in vision. Computer Vision, Graphics, \& Image Processing, 31, 156-177.

Treisman, A. M., \& Gelade, G. (1980). A feature-integration theory of attention. Cognitive Psychology, 12, 97-136.

Treisman, A. M., \& Gormican, S. (1988). Feature analysis in early vision: Evidence from search asymmetries. Psychological Review, 95, $15-48$

Treisman, A., \& Souther, J. (1985). Search asymmetry: A diagnostic for preattentive processing of separable features. Journal of Experimental Psychology: General, 114, 285-310.

Vogels, R., \& Orban, G. A. (1985). The effect of practice on the oblique effect in line orientation judgments. Vision Research, 25, 1679-1687.

Wagemans, J. (1993). Skewed symmetry: A nonaccidental property used to perceive visual forms. Journal of Experimental Psychology: Human Perception \& Performance, 19, 364-380.

Wagemans, J., Van Gool, L., \& D'Ydewalle, G. (1992). Orientational effects and component processes in symmetry detection. Quarterly Journal of Experimental Psychology, 44A, 475-508.

Wenderoth, P. (1994). The salience of vertical symmetry. Perception, 23, 221-236.

Westheimer, G., \& LI, W. (1996). Classifying illusory contours by means of orientation discrimination. Journal of Neurophysiology, $\mathbf{7 5}$, 523-528.

West LAND, S., \& Foster, D. H. (1995). Optimized model of orientationline-target detection using vertical and horizontal filters. Journal of the Optical Society of America A, 12, 1617-1622.

Wolfe, J. M. (1994). Guided Search 2.0: A revised model of visual search. Psychonomic Bulletin \& Review, 1, 202-238.

Wolfe, J. M. (1998). Visual search. In H. Pashler (Ed.), Visual attention (pp. 13-73). Hove, U.K.: Psychology Press.

\section{NOTES}

1. It should be noted that the present experiments were completed before we learned about Found and Müller's (1997) study.

2. It is not clear which axis would be used to describe the orientation of the asymmetrical shape. The oblique axis proposed here is only one of several possible axes. The preference for this axis, however, could be based on a different interpretation of the shape. For example, the asymmetrical shape could be perceived as a near symmetrical surface tilted 
in depth, with the oblique axis being an axis of skewed symmetry (cf. Wagemans, 1993). In any case, further manipulations would be required to examine the choice of alternative axes (e.g., by changing the orientation of the shape in order to bring the putative axis into alignment with the vertical). The main point is that the absence of an orientation asymmetry for the asymmetrical shape suggests that the detection of its orientation was based on a global rather than local analysis.

3. As was noted in the General Method section, the elongated curve also differed from the elongated polygon by its aspect ratio: The curve had an aspect ratio of 2 (or, a length:width ratio of 6:3), whereas the polygon had an aspect ratio of 1.5 (a length:width ratio of $6: 4$ ). Could this relatively minor enhancement in aspect ratio account for the dramatic reversal in the orientation asymmetry? We suggest not. First, both the elongated curves and the elongated polygons had the same length; the increased aspect ratio of the curve was due to its smaller width. It seems unlikely that this difference in width could have caused the reversal in orientation asymmetry. Second, as we noted earlier, Quinlan and Humphreys (1993) observed, for the elongated shape under aspect ratios ranging from $6: 5$ to $6: 3$, that participants never consistently chose the axis of elongation as the principal axis of this shape: Increasing the aspect ratio in order to enhance elongation did not affect participants' preferences. This further suggests that the aspect ratio by itself was not an important factor.

(Manuscript received January 22, 1999; revision accepted for publication August 24, 2000.) 